\title{
An Eco-Epidemiological Predator-Prey Model where Predators Distinguish Between Susceptible and Infected Prey
}

\author{
David Greenhalgh ${ }^{(1)}$, Qamar J. A. Khan ${ }^{(2)}$ and Joseph S. Pettigrew ${ }^{(1)}$ \\ (1) Department of Mathematics and Statistics, \\ University of Strathclyde, Glasgow, U.K. \\ (2) Department of Mathematics and Statistics, \\ College of Science, Sultan Qaboos University, \\ Muscat, Sultanate of Oman
}

\begin{abstract}
A predator-prey model with disease amongst the prey and ratio-dependent functional response for both infected and susceptible prey is proposed and its features analysed. This work is based on previous mathematical models to analyse the important ecosystem of the Salton Sea in Southern California and New Mexico where birds (particularly pelicans) prey on fish (particularly tilapia). The dynamics of the system around each of the ecologically meaningful equilibria are presented. Natural disease control is considered before studying the impact of the disease in the absence of predators, the interaction of predators and healthy prey and the disease effects on predators in the absence of healthy prey. Our theoretical results are confirmed by numerical simulation.
\end{abstract}

Keywords: Epidemiology, ecology, differential equations, equilibrium, stability, Hopf bifurcation.

\section{Introduction}

The Salton Sea in the desert of Southern California, New Mexico is an important eco-epidemiological system where birds (particularly pelicans) prey on fish (particularly tilapia). Over the past few years there have been large-scale bird mortalities at the sea with thousands of pelicans dying from avian botulism. The vibrio class of bacteria is very common in salt water fish and those infected with vibrio may have salt water present in their tissue. As the fish struggle in their death process they tend to rise to the surface of the sea for oxygen. When they do they become very attractive to fish-eating birds, specifically the 
pelicans. When pelicans and other fish-eating birds eat vibrio infected fish alive a large number become infected with botulism and die off.

In this paper we shall study a predator-prey model where disease spreads amongst the prey with (different) ratio-dependent functional responses for both infected and susceptible prey. This work is based upon previous mathematical models of the Salton Sea.

There is a large literature already existing on the subject of ecological systems under the influence of epidemiological factors. Anderson and May [1] studied five prey-predator, parasite interactions where parasites infect either the prey or the predator. Hadeler and Freedman [2] described a predator-prey model where the prey is infected by a parasite and in turn infects the predator with that parasite. Gulland [3] described a number of possible situations in which predators may be affected by various diseases. Haque and Venturino [4] proposed a predator-prey model with an epidemic spreading only among the predators by contact between a well individual and an infected one.

There are two types of prey-predator interaction models in the literature: the first is where prey eaten per predator per unit time is a function of the prey density and in the second type (called predator-dependent functional response) this is a function of both prey and predator. Ratio-dependent functional response is a subtype of predator-dependent functional response and assumes that the prey eaten per unit time is a function of the ratio of prey to predator. Ratio-dependent predator-prey models are more appropriate for predator-prey interaction where predation involves serious searching, for example, predator animals searching for prey in Kuang [5]. It is also a fact that ratio-dependent models are more flexible and versatile. Various field study and laboratory experiments support ratio-dependent predator-prey models, for example [6], [7] and $[8]$.

Chattopadhyay and Bairagi [9], and Sarkar et al. [10] studied tilapia and pelican interaction models for the Salton Sea. In both models the authors assumed that due to infection, tilapia become weak and pelicans interact only with infected tilapia. Later using the same viewpoint Greenhalgh and Haque [11] described a ratio-dependent predator-prey interaction model where susceptibles experienced no predation. This is unlikely to be realistic for most species. Chattopadhyay et al. [12] modified the model discussed in [9] by introducing interaction of pelicans with susceptible fish and assumed that feeding on infected fish increases the death rate of the pelicans. Here we modify the ratio-dependent model of [11] and consider that the susceptible and the infected populations are exposed to the predator to varying degrees. However, the predator preys preferentially on the most numerous prey type.

\section{The Mathematical Model}

We consider here a predator-prey model, in which an epidemic spreads in the prey. We are thinking of predators being pelicans and prey tilapia. We assume that the disease is transmitted by direct contact among the prey. In the presence 
of vibrio infection the prey population is divided into two classes, namely susceptible tilapia, denoted by $S(t)$, and infected tilapia, denoted by $I(t)$. Therefore at time $t$ the total tilapia population is

$$
X(t)=S(t)+I(t) .
$$

We assume that only susceptible tilapia breed and that, in the absence of infection, population growth is given by a logistic function with carrying capacity $k$. Both susceptible and infected tilapia are subject to predation by the pelicans. The pelicans preferentially eat infected tilapia because the tilapia become weak due to infection and rise to the surface of the sea for oxygen. As they are relatively easy to catch, these infected tilapia become more attractive to pelicans.

With the above assumptions the basic equations of the model are

$$
\begin{aligned}
\frac{d S}{d t} & =r S\left(1-\frac{S+I}{k}\right)-\lambda S I-\frac{p Y S}{m Y+S} \\
\frac{d I}{d t} & =\lambda S I-\frac{c Y I}{m Y+I}-\gamma I \\
\frac{d Y}{d t} & =\delta Y\left(1-\frac{h Y}{I+S}\right) .
\end{aligned}
$$

Here the parameters are as follows:

$$
\begin{array}{ll}
r & \text { the species growth rate of tilapia in the breeding subpopulation, } \\
\lambda & \text { the disease transmission coefficient, } \\
\gamma & \text { the per capita death rate of infected prey, } \\
p \text { and } c & \begin{array}{l}
\text { search rate of the pelicans towards susceptible and infected tilapia, } \\
\text { respectively, }
\end{array} \\
\delta & \text { the per capita growth rate of the pelicans, } \\
h & \begin{array}{l}
\text { a constant relating to the density dependent mortality of the preda- } \\
\text { tor population, }
\end{array} \\
m & \text { a strictly positive constant. }
\end{array}
$$

In order to reduce the number of parameters, we define $\tau=\lambda t, r_{1}=\frac{r}{\lambda}$, $p_{1}=\frac{p}{\lambda}, \gamma_{1}=\frac{\gamma}{\lambda}, \delta_{1}=\frac{\delta}{\lambda}$ and $c_{1}=\frac{c}{\lambda}$. Then choosing the new parameters and renaming $\tau$ as $t$, we find that the system is

$$
\begin{aligned}
\frac{d S}{d t} & =r_{1} S\left(1-\frac{S+I}{k}\right)-S I-\frac{p_{1} Y S}{m Y+S} \\
\frac{d I}{d t} & =S I-\frac{c_{1} Y I}{m Y+I}-\gamma_{1} I \\
\frac{d Y}{d t} & =\delta_{1} Y\left(1-\frac{h Y}{I+S}\right) .
\end{aligned}
$$

It is straightforward to show that the solutions to these equations are always positive. 
We assume that all the parameters in the model are strictly positive and that $S(0) \geq 0, I(0) \geq 0$ and $Y(0) \geq 0$. As

$$
0 \leq \frac{S}{m Y+S} \leq 1, \quad 0<\frac{p_{1} Y S}{m Y+S} \leq p_{1} Y .
$$

Hence it is natural to interpret this item as zero when $S=Y=0$. Similarly it is natural to interpret the term

$$
\frac{c_{1} Y I}{m Y+I}
$$

as zero when $Y=I=0$.

If $I(0)=S(0)=0$ and $Y(0) \geq 0$, then (2.1)(iii) is interpreted as implying that $Y(t)=0$ for all $t$. It is straightforward to show that the solution to these equations are always positive and $S(0)>0$ implies that $S(t)>0$ for all $t$, $I(0)>0$ implies that $I(t)>0$ for all $t$ and $Y(0)>0$ and at least one of $S(0)$ and $I(0)$ are strictly positive implies that $Y(t)>0$ for all $t$.

Leslie $[13,14]$ introduced the following mathematical model for a predatorprey system where $X$ is the prey and $Y$ is the predator

$$
\begin{aligned}
& \frac{d X}{d t}=\left(r_{1}-b X\right) X-p(X) Y, \\
& \frac{d Y}{d t}=\left(r_{2}-\frac{a_{2} Y}{X}\right) Y .
\end{aligned}
$$

$\frac{Y}{X}$ is the Leslie-Gower term which measures the loss in the predator population due to the relative scarcity of the prey. $p(X)$ corresponds to the per capita rate at which predators consume prey and $r_{1}, r_{2}, b$ and $a_{2}$ are constants. Thus our model is based on the Leslie-Gower model.

There are several previous papers concerning Leslie-Gower eco-epidemiological models with ratio-dependent and prey-dependent functional response. Here we shall list some of them and differences between our model and theirs. The study of eco-epidemiology was started in 1999 by Chattopadhyay and Arino [15] who studied the following system of differential equations:

$$
\begin{aligned}
\frac{d S}{d t} & =r(S+I)\left(1-\frac{S+I}{k}\right)-b S I-\eta \gamma_{1}(S) Y, \\
\frac{d I}{d t} & =b S I-\gamma(I) Y-c I, \\
\frac{d Y}{d t} & \left.=\varepsilon \gamma(I)+\eta \varepsilon \gamma_{1}(S)-d\right) Y,
\end{aligned}
$$

where $\gamma(I)$ and $\eta \gamma_{1}(S)$ are the predator response functions, $k$ is the carrying capacity of the environment for prey and $r, b, c$ and $d$ are constants. This differs from our model in that firstly both infected and susceptible prey contribute to prey growth and secondly the predator equation is different. Haque and Chattopadhyay [16] and later Jin and Haque [17] study an eco-epidemiological 
model for the Salton Sea with disease in the prey. This model differs from ours in that the predator consumes only infected prey not both types of prey and also the predator response function is not ratio-dependent. Also the disease transmission term is a generalisation of ours to $\lambda I^{p} S^{q}$ where $p$ and $q$ are positive integers and again the predator equation is different.

Arino et al. [18] discuss a ratio-dependent predator-prey model with disease in the prey. This has some similarities with our model but also some differences. Firstly in the logistic term for the growth of the susceptible prey population only deaths of susceptible prey are taken into account whereas in our model there are deaths of both susceptible and infected prey. Secondly in the functional response terms for both susceptible and infected prey the denominators in the model of Arino et al. are of the form (using our notation) $m Y+S+I$, whereas in our model they are $m Y+S$ and $m Y+I$ respectively. Thirdly there are differences in the predator growth equation which has predator growth terms corresponding to consumption of susceptible and infected prey and a linear death term.

Kundu and Chattopadhyay [19] study a model for a predator-prey system with disease amongst the prey which was similar to ours but the predator response to infected prey is simply a function of the number of infected prey and the predator growth equation is not a Leslie-Gower one.

Greenhalgh and Haque [11] study a ratio-dependent predator-prey model with disease in the prey but this differs from the current model as follows: Firstly the growth function of the susceptible prey is purely logistic, depending only on the susceptible prey, whereas in the current model the infected prey also indirectly affects the susceptible prey growth rate. Secondly in [11] the predator preys only on infected prey whereas in the current model the predator preys on both susceptible and infected prey. Thirdly the predator carrying capacity depends only on the infected prey whereas in the current model it depends on the total number of prey.

Xaio and Chen [20] also study a ratio-dependent predator-prey model with disease in the prey but in their model the predators do not consume susceptible prey and the predator growth equation is different and is not of Leslie-Gower type.

Pal and Samanta [21] study a predator-prey model incorporating a prey refuge with disease in the prey. The paper extends the model of Xaio and Chen [20]. In a later paper, Pal and Samanta [22] they modify this model in an arguably more realistic fashion by taking the prey disease transmission term to be frequency dependent $\frac{a S I}{S+I}$ rather than density dependent $a S I$. Thus in the frequency dependent model the per capita disease contact rate is constant.

Wang and Feng [23] also extend the model of Xaio and Chen [20] including stage structure into the predator population and a time delay due to the gestation of the predator into the system. However again in all three models unlike our current model the predators do not consume infected prey and the predator growth equation is not the same as ours.

Rahman and Chakravarty [24] discuss a predator-prey model with disease in the prey in which the functional response to susceptible and infected prey are 
respectively $\frac{c_{1} S Y}{a+(S+I) Y}$ and $\frac{c_{2} I Y}{a+(S+I) Y}$ where $a, c_{1}$ and $c_{2}$ are constants. This is a modification of a model studied by Cosner et al. [25]. This model differs from ours in that both the per capita predation rates of susceptible and infected prey have different functional forms and yet again the predator growth equation is not of Leslie-Gower type.

There are many other papers which consider eco-epidemiological models with disease in the prey (or the predator), for example Venturino [26, 27], Haque and Venturino [4, 28], Chattopadhyay and Bairagi [9], Xaio and Chen [29, 30], Chattopadhyay, Srinivasu and Bairagi [12] and Mukhopadhyay and Bhattacharya [31] amongst others but we believe that the models which we have surveyed above are the most relevant to our study.

\section{Boundedness Results}

We have three results on the boundedness of the system (2.1). First of all, we consider the prey.

Proposition 1 The prey are always bounded above.

Proof. If $S(0)=0$ then the result is trivial. If $S(0)>0$ then $S(t)>0$ for all $t$. On adding equations (2.1)(i) and (2.1)(ii), we get

$$
\frac{d S}{d t}+\frac{d I}{d t} \leq r_{1} S\left(1-\frac{S+I}{k}\right)
$$

It follows then that $\lim \sup _{t \rightarrow \infty}(S(t)+I(t)) \leq k$.

Proposition 2 The number of predators $Y$ is always bounded above.

Proof. If $Y(0)=0$ the result is obvious. If $Y(0)>0$, then using equation (2.1)(iii) we see that

$$
\frac{d Y}{d t}<0 \text { if } \frac{h Y}{I+S}>1 .
$$

Suppose that $\limsup _{t \rightarrow \infty} Y>\frac{k}{h}$. i.e. $\limsup _{t \rightarrow \infty} Y>\frac{k+\epsilon}{h}$ for some $\epsilon>0$. Hence $Y-\frac{k+\epsilon}{h}>0$ in infinitely many disjoint intervals $\left(t_{0}, t_{1}\right),\left(t_{2}, t_{3}\right),\left(t_{4}, t_{5}\right), \ldots$ and outside these intervals $Y-\frac{k+\epsilon}{h} \leq 0$.

As limsup $\sup _{t \rightarrow \infty}(I+S) \leq k$ without loss of generality we can assume that in these intervals $\frac{k+\epsilon}{h}>\frac{I+S}{h}$ so $\frac{d Y}{d t}<0$ in these intervals. If one such interval is $\left(t_{2 r}, t_{2 r+1}\right), r$ an integer, then for $t \in\left(t_{2 r}, t_{2 r+1}\right)$

$$
Y(t) \leq Y\left(t_{2 r}\right)=\frac{k+\epsilon}{h} .
$$

Hence $\lim \sup _{t \rightarrow \infty} Y(t) \leq \frac{k+\epsilon}{h}$. As $\epsilon>0$ is arbitrary we deduce that $\lim \sup _{t \rightarrow \infty} Y(t) \leq$ $\frac{k}{h}$.

Proposition 3 The trajectories of system (2.1) are bounded. 
Proof. Define the function $\ell=S+I+Y$ and take its time derivative along the solution of $(2.1)$

$$
\frac{d \ell}{d t}=\frac{d S}{d t}+\frac{d I}{d t}+\frac{d Y}{d t} .
$$

Now

$$
\begin{aligned}
\frac{d \ell}{d t}+q \ell= & r_{1} S-r_{1} S\left(\frac{S+I}{k}\right)-\frac{p_{1} Y S}{m Y+S}-\frac{c_{1} Y I}{m Y+I} \\
& -\gamma_{1} I+\delta_{1} Y-\frac{\delta_{1} h Y^{2}}{I+S}+q S+q I+q Y, \\
= & \left(q+r_{1}\right) S+\left(q+\delta_{1}\right) Y-\left(\gamma_{1}-q\right) I-r_{1} S\left(\frac{S+I}{k}\right) \\
& -\frac{p_{1} Y S}{m Y+S}-\frac{c_{1} Y I}{m Y+I}-\frac{\delta_{1} h Y^{2}}{I+S},
\end{aligned}
$$

where $q$ is a positive constant. For $q<\gamma_{1}$ given $\epsilon>0$ there exists $t_{0}$ such that for $t \geq t_{0}$

$$
\frac{d \ell}{d t}+q \ell \leq m+\epsilon
$$

where $m=\left(q+r_{1}\right) k+\left(q+\delta_{1}\right) \frac{k}{h}$. Hence

$$
\frac{d}{d t}\left(\ell e^{q t}\right) \leq(m+\epsilon) e^{q t}
$$

so

$$
\ell(t) \leq \ell\left(t_{0}\right) e^{-q\left(t-t_{0}\right)}+\frac{m+\epsilon}{q}\left(1-e^{-q\left(t-t_{0}\right)}\right) .
$$

Letting $t \rightarrow \infty$, then letting $\epsilon \rightarrow 0$

$$
\limsup _{t \rightarrow \infty} \ell(t) \leq \frac{m}{q}
$$

independently of the initial conditions.

\section{The Equilibria}

We now examine the equilibria of the system (2.1). We have the following theorem:

Theorem 4 The ecologically meaningful possible equilibria of system (2.1) are

(i) $\bar{E}_{0}=(0,0,0)$, where all populations are extinct, which always exists.

(ii) $\bar{E}_{1}=(k, 0,0)$ where there is only susceptible prey which always exists.

(iii) $\bar{E}_{2}=\left(h Y_{2}, 0, Y_{2}\right)$ where $Y_{2}=\left(r_{1}-\frac{p_{1}}{m+h}\right) \frac{k}{r_{1} h}$. In this equilibrium there are only susceptible prey and predators. This equilibrium always exists if $r_{1}>\frac{p_{1}}{m+h}$. 
(iv) $\bar{E}_{3}=\left(\gamma_{1}, \frac{r_{1}\left(k-\gamma_{1}\right)}{k+r_{1}}, 0\right)$. This equilibrium has only infected and susceptible prey and no predators. It is possible if $k>\gamma_{1}$.

(v) Up to two co-existence equilibria $\bar{E}_{4}, \bar{E}_{5}$. These are given by $\bar{E}_{i}=((h-$ $\left.\left.\bar{x}_{i}\right) \bar{y}, \bar{x}_{i} \bar{y}, \bar{y}\right)$, for $i=4,5$ where $\bar{x}_{i}$ is the $i$ th root of the cubic equation

$$
f(x)=a_{0} x^{3}+a_{1} x^{2}+a_{2} x+a_{3}=0
$$

$$
\text { in } \begin{aligned}
{\left[0, \min \left[h, m+h-\frac{p_{1}}{r_{1}}\right]\right] \text { where } } \\
a_{0}=k\left(r_{1}+\gamma_{1}\right), \\
a_{1}=k\left(p_{1}+c_{1}\right)-2 k r_{1} h-\gamma_{1} h\left(k-r_{1}\right), \\
a_{2}=k r_{1}\left(h^{2}-m^{2}-h m\right)-k p_{1}(h-m)-c_{1}\left[k(m+h)-r_{1} h\right] \\
\quad-\gamma_{1} k m(m+h)-\gamma_{1} r_{1} h^{2}, \\
a_{3}=(m+h) r_{1} h\left(k m-c_{1}-\gamma_{1} m-\frac{p_{1} m k}{r_{1}(m+h)}\right) .
\end{aligned}
$$

Here

$$
\bar{y}=\frac{c_{1}+\gamma_{1}\left(m+x_{i}\right)}{\left(m+x_{i}\right)\left(h-x_{i}\right)}, \quad \text { for } i=4,5 .
$$

Define

$$
\Delta=\frac{4}{27} a_{2}^{3}-\frac{1}{27} a_{1}^{2} a_{2}^{2}+\frac{4}{27} a_{1}^{3} a_{3}-\frac{2}{3} a_{1} a_{2} a_{3}+a_{3}^{2} .
$$

(a) For $\frac{p_{1}}{r_{1}} \geq m+h$, there are no feasible co-existence equilibria.

(b) For $\frac{p_{1}}{r_{1}}<m+h$ and $k m>c_{1}+\gamma_{1} m+\frac{p_{1} m k}{r_{1}(m+h)}$, the cubic equation (4.1) has exactly one real root in the given interval.

(c) Suppose that $\frac{p_{1}}{r_{1}}<m+h$ and $k m<c_{1}+\gamma_{1} m+\frac{p_{1} m k}{r_{1}(m+h)}$.

For $a_{1}^{2}>3 a_{0} a_{2}, a_{1}<0, a_{2}>0$ and $\Delta<0$, the cubic equation (4.1) has two real positive turning points at $\alpha_{1}, \beta_{1}\left(\alpha_{1}<\beta_{1}\right)$, the roots of

$$
3 a_{0} x^{2}+2 a_{1} x+a_{2}=0 .
$$

If

$$
\alpha_{1}<\min \left[h, m+h-\frac{p_{1}}{r_{1}}\right],
$$

then there are two strictly positive real roots of (4.1) in the interval.

If either (i) $a_{1}^{2} \leq 3 a_{0} a_{2}$, (ii) $a_{1} \geq 0$, (iii) $a_{2} \leq 0$, (iv) $\Delta>0$ or (v) $a_{1}^{2}>3 a_{0} a_{2}, a_{1}<0 a_{2}>0, \Delta \geq 0$ and

$$
\alpha_{1} \geq \min \left[h, m+h-\frac{p_{1}}{r_{1}}\right]
$$


then there are no strictly positive real roots of (4.1) in the interval.

If $a_{1}^{2}>3 a_{0} a_{2}, a_{1}<0, a_{2}>0, \Delta=0$ and

$$
\alpha_{1}<\min \left[h, m+h-\frac{p_{1}}{r_{1}}\right]
$$

then (4.1) has one strictly positive repeated real root in the interval.

(d) Suppose that $\frac{p_{1}}{r_{1}}<m+h$ and $k m=c_{1}+\gamma_{1} m+\frac{p_{1} m k}{r_{1}(m+h)}$.

For $a_{1}^{2}>3 a_{0} a_{2}, a_{1}<0, a_{2}>0$ and $\Delta<0$ if

$$
\alpha_{1}<\min \left[h, m+h-\frac{p_{1}}{r_{1}}\right]
$$

then there is exactly one strictly positive real root of (4.1) in the interval $\left(0, \min \left[h, m+h-\frac{p_{1}}{r_{1}}\right]\right)$.

If either (i) $a_{1}^{2} \leq 3 a_{0} a_{2}$, (ii) $a_{1} \geq 0$, (iii) $a_{2} \leq 0$ or (iv) $\Delta \geq 0$ then there are no strictly positive real roots of (4.1) in this interval.

\section{Ecological Interpretation of These Conditions.}

The condition for the existence of $\bar{E}_{2}$ is $r_{1}>\frac{p_{1}}{m+h}$, equivalently $r>\frac{p}{m+h}$. This means that both $r$, the per capita growth rate of the tilapia and $h$, the density-dependent mortality constant are relatively large compared with $p$, the search rate of the pelicans toward infected tilapia.

The condition for the existence of $\bar{E}_{3}$ is $k \lambda>\gamma$. This means that the product of the carrying capacity $k$ of the environment for prey and the disease transmission coefficient $\lambda$ exceeds $\gamma$ the per capita death rate of infected prey. Provided that $r_{1}>\frac{p_{1}}{m+h}$, the condition in Theorem 4(v)(b) for the existence of one or more co-existence equilibria is

$$
\left(1-\frac{p}{(m+h) r}\right) \lambda>\frac{1}{m k}(c+\gamma m)
$$

so the weighted sum of the search rate $c$ of the pelicans towards infected tilapia and the per capita death rate of infected tilapia $\gamma$ is less than the disease transmission coefficient $\lambda$ multiplied by the carrying capacity of the environment for prey, $k$, is less than a term that is related to how strongly the first condition

is satisfied.

$$
r_{1}>\frac{p_{1}}{m+h}
$$

Proof. From equations $(2.1)$ we see that $(0,0,0)$ is always a solution. Moreover for any feasible solution 
(1) Either $S^{*}=0$ or $r_{1}\left(1-\frac{S^{*}+I^{*}}{k}\right)-I^{*}-\frac{p_{1} Y^{*}}{m Y^{*}+S^{*}}=0$,

(2) Either $I^{*}=0$ or $S^{*}-\frac{c_{1} Y^{*}}{m Y^{*}+I^{*}}-\gamma_{1}=0$,

(3) Either $Y^{*}=0$ or $I^{*}+S^{*}=h Y^{*}$.

(i) If $S^{*}=0$ then $I^{*}=0$ and $Y^{*}=0$.

(ii) and (iii) If $S^{*} \neq 0$ and $I^{*}=0$, either (ii) $Y^{*}=0$ which implies that $S^{*}=k$, leading to the equilibrium $\bar{E}_{1}$ or (iii) $Y^{*} \neq 0$ which implies that

$$
r_{1}=\frac{r_{1} S^{*}}{k}+\frac{p_{1} Y^{*}}{m Y^{*}+S^{*}} \text { and } S^{*}=h Y^{*} .
$$

Hence

so

$$
r_{1}=\frac{r_{1} h Y^{*}}{k}+\frac{p_{1}}{m+h},
$$

$$
Y^{*}=\left(r_{1}-\frac{p_{1}}{m+h}\right) \frac{k}{r_{1} h}, \quad S^{*}=\left(r_{1}-\frac{p_{1}}{m+h}\right) \frac{k}{r_{1}} .
$$

This equilibrium $\bar{E}_{2}$ will be feasible if $r_{1}(m+h)>p_{1}$.

(iv) If $S^{*} \neq 0, I^{*} \neq 0$ and $Y^{*}=0$ then $S^{*} I^{*}=\gamma_{1} I^{*}$ so $S^{*}=\gamma_{1}$ which implies that

$$
\begin{aligned}
r_{1}\left(1-\frac{S^{*}+I^{*}}{k}\right)-I^{*} & =0 \\
I^{*}\left(1+\frac{r_{1}}{k}\right) & =r_{1}\left(1-\frac{\gamma_{1}}{k}\right), \\
I^{*} & =\frac{r_{1}\left(k-\gamma_{1}\right)}{k+r_{1}} .
\end{aligned}
$$

Thus we have $\bar{E}_{3}$ which is feasible if $k>\gamma_{1}$.

(v) Finally for a co-existence equilibrium we have $S^{*} \neq 0, I^{*} \neq 0, Y^{*} \neq 0$.

Hence

$$
\begin{aligned}
r_{1}\left(1-\frac{S^{*}+I^{*}}{k}\right)-I^{*}-\frac{p_{1} Y^{*}}{m Y^{*}+S^{*}} & =0, \\
S^{*}-\frac{c_{1} Y^{*}}{m Y^{*}+I^{*}}-\gamma_{1} & =0, \\
I^{*}+S^{*} & =h Y^{*} .
\end{aligned}
$$

Write $x^{*} Y^{*}=I^{*}$

$$
\begin{aligned}
\left(h-x^{*}\right) Y^{*} & =\frac{c_{1} Y^{*}}{m Y^{*}+x^{*} Y^{*}}+\gamma_{1}, \\
& =\frac{c_{1}}{m+x^{*}}+\gamma_{1} \\
& =\frac{c_{1}+\gamma_{1}\left(m+x^{*}\right)}{m+x^{*}} .
\end{aligned}
$$


So

$$
Y^{*}=\frac{\left(c_{1}+\gamma_{1} m\right)+\gamma_{1} x^{*}}{\left(h-x^{*}\right)\left(m+x^{*}\right)} .
$$

Now from equation (4.2) we deduce that

$$
r_{1}\left(1-\frac{h Y^{*}}{k}\right)-x^{*} Y^{*}-\frac{p_{1}}{m+h-x^{*}}=0 .
$$

Thus

$$
Y^{*}=\left(\frac{r_{1}\left(m+h-x^{*}\right)-p_{1}}{m+h-x^{*}}\right)\left(\frac{k}{k x^{*}+r_{1} h}\right) .
$$

For a feasible equilibrium we need $0<x^{*}<h$ and $x^{*}<\left(m+h-\frac{p_{1}}{r_{1}}\right)$ also

$$
\frac{\left(c_{1}+\gamma_{1} m\right)+\gamma_{1} x^{*}}{\left(h-x^{*}\right)\left(m+x^{*}\right)}=\left(\frac{r_{1}\left(m+h-x^{*}\right)-p_{1}}{\left(m+h-x^{*}\right)}\right)\left(\frac{k}{k x^{*}+r_{1} h}\right)
$$

i.e.

$$
\begin{aligned}
& -\left(\left(c_{1}+\gamma_{1} m\right)+\gamma_{1} x^{*}\right)\left(m+h-x^{*}\right)\left(k x^{*}+r_{1} h\right) \\
& \quad+k\left(h-x^{*}\right)\left(m+x^{*}\right)\left(r_{1}\left(m+h-x^{*}\right)-p_{1}\right)=0 .
\end{aligned}
$$

Expanding this expression we can see that it is the cubic (4.1)

$$
a_{0} x^{* 3}+a_{1} x^{* 2}+a_{2} x^{*}+a_{3}=0
$$

described earlier.

(a) Clearly for $\frac{p_{1}}{r_{1}} \geq m+h$ there are no feasible co-existence equilibria.

(b) For $k m>c_{1}+\gamma_{1} m+\frac{p_{1} m k}{r_{1}(m+h)}, a_{3}>0$.

Using (4.4), $f(h)=-\left(\left(c_{1}+\gamma_{1} m\right)+\gamma_{1} h\right) m\left(k+r_{1}\right) h<0$.

If $x=m+h-\frac{p_{1}}{r_{1}}<h$ (note that $m+h>\frac{p_{1}}{r_{1}}$ ), again using (4.4) we deduce that

$$
f\left(m+h-\frac{p_{1}}{r_{1}}\right)=-\left(\left(c_{1}+\gamma_{1} m\right)+\gamma_{1} x\right) \frac{p_{1}}{r_{1}}\left(k x+r_{1} h\right)<0 .
$$

Therefore $f\left(\min \left[h, m+h-\frac{p_{1}}{r_{1}}\right]\right)<0$. Hence the cubic equation (4.1) has one or three real roots in the interval $\left[0, \min \left[h, m+h-\frac{p_{1}}{r_{1}}\right]\right]$. But it has one real root in $\left[\min \left[h, m+h-\frac{p_{1}}{r_{1}}\right], \infty\right]$. Therefore it has exactly one real root in the interval $\left[0, \min \left[h, m+h-\frac{p_{1}}{r_{1}}\right]\right]$ in this case. 
(c) For $\frac{p_{1}}{r_{1}}<m+h$ and $k m<c_{1}+\gamma_{1} m+\frac{p_{1} m k}{r_{1}(m+h)}, a_{3}<0$ and as above $f\left(\min \left[h, m+h-\frac{p_{1}}{r_{1}}\right]\right)<0$. Hence the cubic equation (4.1) has either zero or two positive real roots in the interval. The derivative of $f(x)$ given by $(4.1)$ is

$$
g(x)=3 a_{0} x^{2}+2 a_{1} x+a_{2} .
$$

The turning points are the roots $\alpha_{1}, \beta_{1}$ of $g(x)=0$ and $\Delta=f\left(\alpha_{1}\right) f\left(\beta_{1}\right)$ [32]. If $a_{1}^{2}>3 a_{0} a_{2}, a_{1}<0, a_{2}>0$ and $\Delta<0$, the cubic equation (4.1) has two real turning points at $\alpha_{1}, \beta_{1}$ where $0 \leq \alpha_{1}<\beta_{1}$. If

$$
\alpha_{1}<\min \left[h, m+h-\frac{p_{1}}{r_{1}}\right]
$$

then by considering the shape of $f(x)$ we see that there are two strictly positive real roots of (4.1) in the interval.

If $a_{1}^{2}>3 a_{0} a_{2}, a_{1}<0, a_{2}>0, \Delta=0$ and

$$
\alpha_{1}<\min \left[h, m+h-\frac{p_{1}}{r_{1}}\right]
$$

then $f(x)$ has one repeated strictly positive real root in the interval. If either $a_{1}^{2} \leq 3 a_{0} a_{2}$, or $a_{1} \geq 0$ or $a_{2} \leq 0$ or $\Delta>0$ then $f(x)$ has no strictly positive real roots in the interval.

\subsection{The Case $p_{1}=0$}

We can gain further insight into when what number of equilibria are possible by considering the special case $p_{1}=0$. When $p_{1}=0$ we need $0<x^{*}<h$ for a feasible equilibrium. The cubic (4.4) reduces to $x^{*}=m+h$ (infeasible) or

$$
-k\left(h-x^{*}\right)\left(m+x^{*}\right) r_{1}+\left(\left(c_{1}+\gamma_{1} m\right)+\gamma_{1} x^{*}\right)\left(k x^{*}+r_{1} h\right)=0 .
$$

This is a quadratic

$$
\ell\left(x^{*}\right)=b_{0} x^{* 2}+b_{1} x^{*}+b_{2}=0
$$

where

$$
\begin{aligned}
& b_{0}=k\left(\gamma_{1}+r_{1}\right), \\
& b_{1}=k\left(c_{1}+\gamma_{1} m\right)+r_{1} h \gamma_{1}+k m r_{1}-k h r_{1}, \\
& b_{2}=\left(c_{1}+\gamma_{1} m\right) r_{1} h-k h r_{1} m .
\end{aligned}
$$

(i) Note that $\ell(h)>0$. Hence if $c_{1}+\gamma_{1} m<k m$ there is exactly one root of $\ell\left(x^{*}\right)=0$ in $[0, h]$. 
(ii) If $c_{1}+\gamma_{1} m>\max \left[k m, h r_{1}\right]$ then $b_{1}>0, b_{2}>0$, so $\ell\left(x^{*}\right)=0$ has no real roots in $[0, h]$.

(iii) To see that it is possible to have two feasible co-existence equilibria it is sufficient to show that it is possible to have two positive real roots of $\ell\left(x^{*}\right)=0$ in $[0, h]$. Choose $\gamma_{1}<k$ and $c_{1}=\left(k-\gamma_{1}\right) m+\epsilon$ where $\epsilon>0$ is very small. Choose $h$ very large so that

$$
b_{1}=k[k m+\epsilon]+r_{1} h \gamma_{1}+k m r_{1}-k h r_{1}<0 .
$$

Then if $h$ is large enough and $\epsilon$ is small enough $b_{1}<0, b_{2}>0$ and $b_{1}^{2}-4 b_{0} b_{2}>0$. So the quadratic

$$
\ell\left(x^{*}\right)=b_{0} x^{* 2}+b_{1} x^{*}+b_{2}
$$

has two positive real roots and is minimised at

$$
x^{*}=-\frac{b_{1}}{2 b_{0}}>0 .
$$

But recalling that $h$ is very large and $\epsilon$ is very small

$$
-\frac{b_{1}}{2 b_{0}} \approx \frac{r_{1} h}{2 k} \frac{\left(k-\gamma_{1}\right)}{\left(\gamma_{1}+r_{1}\right)}<h
$$

as $2\left(\gamma_{1}+r_{1}\right) k>r_{1}\left(k-\gamma_{1}\right)$. Therefore the quadratic has two real roots in $[0, h]$. Hence it is possible for there to be either no, one or two co-existence equilibria.

This concludes our equilibrium analysis of the predator-prey model with disease. In the next section we shall look at the stability of these equilibria.

\section{$5 \quad$ Stability}

At a general equilibrium $(S, I, Y)$ the stability matrix or Jacobian of the system is

$$
\mathbf{J}=\left(\begin{array}{ccc}
r_{1}-\frac{2 r_{1} S}{k}-\frac{r_{1} I}{k}-I-\frac{m p_{1} Y^{2}}{(m Y+S)^{2}} & \frac{-r_{1}}{k} S-S & \frac{-p_{1} S^{2}}{(m Y+S)^{2}} \\
I & S-\gamma_{1}-\frac{c_{1} m Y^{2}}{(m Y+I)^{2}} & \frac{-c_{1} I^{2}}{(m Y+I)^{2}} \\
\frac{\delta_{1} h Y^{2}}{(I+S)^{2}} & \frac{\delta_{1} h Y^{2}}{(I+S)^{2}} & \delta_{1}\left(1-\frac{2 h Y}{I+S}\right)
\end{array}\right) .
$$

\subsection{Dynamics of the System Around $\bar{E}_{0}$}

The stability matrix is not well-defined at the equilibrium $\bar{E}_{0}$. To show that $\bar{E}_{0}$ is unstable it is sufficient to show that not all trajectories starting in a small ball of radius $\epsilon>0$ approach $\bar{E}_{0}$. Consider a trajectory with $Y_{0}=0$ and $S_{0}>0$ then $Y(t)=0$ and $S(t)>0$ for all $t$. 
Hence

$$
\frac{1}{S} \frac{d S}{d t}=r_{1}\left(1-\frac{S+I}{k}\right)-I>\frac{r_{1}}{2}
$$

if $S$ and $I$ are small enough. Hence $S \geq S_{0} e^{\frac{r_{1} t}{2}}$. So this trajectory cannot approach $\bar{E}_{0}$ which is unstable.

\subsection{Dynamics of the System Around $\bar{E}_{1}$}

The stability matrix is also not well defined at the equilibrium $\bar{E}_{1}$. To show that $\bar{E}_{1}$ is unstable suppose that $\bar{E}_{1}$ is locally asymptotically stable (LAS). Now consider a trajectory with $Y(0)>0$ and either $I_{0}>0$, or $S_{0}>0$, hence either $I(t)>0$ for all $t$ or $S(t)>0$ for all $t$. As this trajectory approaches the equilibrium $\bar{E}_{1}$

$$
\frac{d Y}{d t} \rightarrow \delta_{1} Y .
$$

Hence if this trajectory approaches $\bar{E}_{1}$ there is a $t_{0}$ such that for $t \geq t_{0}$

$$
\frac{d Y}{d t} \geq \frac{\delta_{1} Y}{2} .
$$

Also $Y\left(t_{0}\right)>0$ as $Y(0)>0$. So

$$
Y(t) \geq e^{\frac{\delta_{1}\left(t-t_{0}\right)}{2}}
$$

for $t \geq t_{0}$ which is a contradiction. Therefore $\bar{E}_{1}$ is unstable.

\subsection{Dynamics of the System Around $\bar{E}_{2}$}

At the equilibrium $\bar{E}_{2}$ one eigenvalue is $J_{22}=\frac{k}{r_{1}}\left(r_{1}-\frac{p_{1}}{m+h}\right)-\gamma_{1}-\frac{c_{1}}{m}$. The remaining eigenvalues satisfy the characteristic equation

$$
\operatorname{det}\left(\begin{array}{cc}
-r_{1}+\frac{p_{1}(m+2 h)}{(m+h)^{2}}-\omega & -\frac{p_{1} h^{2}}{(m+h)^{2}} \\
\frac{\delta_{1}}{h} & -\delta_{1}-\omega
\end{array}\right)=0 .
$$

This is equivalent to

$$
\omega^{2}+a_{1} \omega+a_{2}=0
$$

where

$$
a_{1}=\delta_{1}+r_{1}-\frac{p_{1}(m+2 h)}{(m+h)^{2}}
$$

and

$$
a_{2}=\frac{\delta_{1} r_{1} h Y_{2}}{k}>0 .
$$

Hence this equilibrium is stable if

$$
\gamma_{1}+\frac{c_{1}}{m}+\frac{k p_{1}}{r_{1}(m+h)}>k
$$


and

$$
\delta_{1}+r_{1}>\frac{p_{1}(m+2 h)}{(m+h)^{2}} .
$$

The equilibrium is unstable if either

$$
k>\gamma_{1}+\frac{c_{1}}{m}+\frac{k p_{1}}{r_{1}(m+h)}
$$

or

$$
\frac{p_{1}(m+2 h)}{(m+h)^{2}}>\delta_{1}+r_{1} .
$$

We have already discussed the biological significance of the first of these stability conditions (5.1). The second condition can be rewritten as

$$
\frac{p}{h}\left(1-\left(\frac{h}{m+h}\right)^{2}\right)>\delta+r .
$$

So this condition will be satisfied if $p$, the search rate of the pelicans towards susceptible tilapia is high, and $h$, the density-dependent mortality rate of the pelicans is small compared with the sum of the per capita birth rates of the pelicans and the tilapia.

\subsection{Dynamics of the System Around $\bar{E}_{3}$}

At the equilibrium $\bar{E}_{3}=\left(S_{3}, I_{3}, 0\right)$ the Jacobian is

$$
\mathbf{J}_{\mathbf{3}}=\left(\begin{array}{ccc}
-\frac{r_{1} S_{3}}{k} & -\left(\frac{r_{1}}{k}+1\right) S_{3} & -p_{1} \\
I_{3} & 0 & -c_{1} \\
0 & 0 & \delta_{1}
\end{array}\right)
$$

One eigenvalue is clearly $\delta_{1}>0$ so this equilibrium is unstable. The other two eigenvalues have negative real parts.

\subsection{Dynamics of the System Around the Co-existence Equi- libria $\bar{E}_{4}$ and $\bar{E}_{5}$}

At the equilibria $\bar{E}_{4}$ and $\bar{E}_{5}=(\bar{S}, \bar{I}, \bar{Y})$ the Jacobian matrix is

$$
\begin{aligned}
\mathbf{J}_{4} & =\left(\begin{array}{ccc}
-\frac{r_{1} \bar{S}}{k}+\frac{p_{1} \bar{Y} \bar{S}}{(m \bar{Y}+\bar{S})^{2}} & -\frac{r_{1} \bar{S}}{k}-\bar{S} & -\frac{p_{1} \bar{S}^{2}}{(m \bar{Y}+\bar{S})^{2}} \\
\bar{I} & \frac{c_{1} \bar{Y} \bar{I}}{(m \bar{Y}+\bar{I})^{2}} & -\frac{c_{1} \bar{I}^{2}}{(m \bar{Y}+\bar{I})^{2}} \\
\frac{\delta_{1}}{h} & \frac{\delta_{1}}{h} & -\delta_{1}
\end{array}\right) \\
& =\left(\begin{array}{ccc}
-a_{11}+a_{12} \bar{Y}-\omega & -a_{11}-\bar{S} & -a_{12} \bar{S} \\
\bar{I} & A-\omega & -\bar{A} x^{*} \\
\frac{\delta_{1}}{h} & \frac{\delta_{1}}{h} & -\delta_{1}-\omega
\end{array}\right)
\end{aligned}
$$


where

$$
a_{11}=\frac{r_{1} \bar{S}}{k}, a_{12}=\frac{p_{1} \bar{S}}{(m \bar{Y}+\bar{S})^{2}} \quad \text { and } \quad A=\frac{c_{1} \bar{Y} \bar{I}}{(m \bar{Y}+\bar{I})^{2}}
$$

The characteristic equation associated with the possible equilibria $\bar{E}_{4}$ and $\bar{E}_{5}$ of this model is

$$
\begin{aligned}
& \left(-\delta_{1}-\omega\right)(A-\omega)\left(-a_{11}+a_{12} \bar{Y}-\omega\right)+\frac{\delta_{1}}{h} A x^{*}\left(a_{11}+\bar{S}\right)-\frac{\delta_{1}}{h} I a_{12} \bar{S} \\
& +a_{12} \bar{S} \frac{\delta_{1}}{h}(A-\omega)+\frac{\delta_{1}}{h} A x^{*}\left(-a_{11}+a_{12} \bar{Y}-\omega\right)-\left(\delta_{1}+\omega\right)\left(a_{11}+\bar{S}\right) I=0 .
\end{aligned}
$$

This can be expressed as a cubic equation

$$
\omega^{3}+e_{1} \omega^{2}+e_{2} \omega+e_{3}=0,
$$

where

$$
\begin{aligned}
e_{1}= & \delta_{1}+a_{11}-a_{12} \bar{Y}-A, \\
e_{2}= & \delta_{1}\left(a_{11}-a_{12} \bar{Y}-A\right)-A\left(a_{11}-a_{12} \bar{Y}\right)+a_{12} \frac{\bar{S} \delta_{1}}{h}+\frac{\delta_{1}}{h} A x^{*}+\left(a_{11}+\bar{S}\right) \bar{I}, \\
e_{3}= & -\delta_{1} A\left(a_{11}-a_{12} \bar{Y}\right)-\frac{\delta_{1}}{h} A x^{*}\left(a_{11}+\bar{S}\right)+\frac{\delta_{1}}{h} \bar{I} a_{12} \bar{S}-\frac{\delta_{1}}{h} A \bar{S} a_{12} \\
& +\frac{\delta_{1}}{h} A x^{*}\left(a_{11}-a_{12} \bar{Y}\right)+\delta_{1}\left(a_{11}+\bar{S}\right) \bar{I} .
\end{aligned}
$$

The Routh-Hurwitz conditions for the characteristic equation to have only roots with strictly negative real parts and hence the co-existence equilibrium to be LAS are $e_{1}>0, e_{3}>0$ and $e_{1} e_{2}>e_{3}$.

If we consider the special case where $p_{1}=c_{1}=0$ then this implies that

$$
\begin{aligned}
& e_{1}=\delta_{1}+a_{11}, \\
& e_{2}=\delta_{1} a_{11}+\left(a_{11}+\bar{S}\right) \bar{I}, \\
& e_{3}=\delta_{1}\left(a_{11}+\bar{S}\right) \bar{I},
\end{aligned}
$$

in this case. So the co-existence equilibrium is LAS when it exists.

Note that equation (4.1) and hence $x^{*}$ does not depend on $\delta_{1}$. Hence it is natural to express $e_{1}, e_{2}$ and $e_{3}$ in terms of $\delta_{1}$ and see what happens as $\delta_{1}$ varies. In terms of $\delta_{1}$

$$
\begin{aligned}
& e_{1}=\delta_{1}+\xi_{1}, \\
& e_{2}=\delta_{1} \xi_{2}+\xi_{3}, \\
& e_{3}=\delta_{1} \xi_{4},
\end{aligned}
$$

where

$$
\begin{aligned}
& \xi_{1}=a_{11}-a_{12} \bar{Y}-A \\
& \xi_{2}=a_{11}-a_{12}\left(\bar{Y}-\frac{\bar{S}}{h}\right)-A\left(1-\frac{x^{*}}{h}\right), \quad \xi_{2}>\xi_{1}, \\
& \xi_{3}=\left(a_{11}+\bar{S}\right) \bar{I}-A\left(a_{11}-a_{12} \bar{Y}\right), \\
& \xi_{4}=-A\left(a_{11}-a_{12} \bar{Y}\right)-\frac{A \bar{S} x^{*}}{h}+\frac{I a_{12} \bar{S}}{h}-\frac{a_{12} \bar{S} A}{h}-\frac{A \bar{Y} x^{*} a_{12}}{h}+\left(a_{11}+\bar{S}\right) \bar{I} .
\end{aligned}
$$


If $\xi_{4}<0$, the equilibrium is unstable for all $\delta_{1}$.

This situation is rather complicated to analyse so we consider the different possibilities depending on the signs of $\xi_{1}, \xi_{2}, \xi_{3}$ and $\xi_{4}$. Examining the RouthHurwitz conditions

$$
e_{1}>0, e_{3}>0 \text { and } e_{1} e_{2}>e_{3}
$$

in terms of $\delta_{1}$, the condition $e_{1}>0$ is given by a straight line of slope one intercept $\xi_{1}$, the condition $e_{3}>0$ by a straight line through the origin and in general apart from degenerate parameter values $e_{1} e_{2}>e_{3}$ by a parabola. Looking at these conditions graphically it is straightforward to show that:

(i) If $\xi_{4}<0$ then any co-existence equilibrium is unstable for all $\delta_{1}$;

(ii) If $\xi_{1}>0, \xi_{3}>0, \xi_{4}>0$ then any co-existence equilibrium is stable in a range such as $\left(0, \delta_{1}^{0}\right),\left(\delta_{1}^{1}, \infty\right)$ (including always stable as a possibility);

(iii) If $\xi_{1} \leq 0, \xi_{2}>0, \xi_{4}>0$ then a co-existence equilibrium is stable in a range such as $\left(\delta_{1}^{0}, \infty\right)$;

(iv) If $\xi_{1} \leq 0, \xi_{2}<0, \xi_{1} \xi_{2}>\xi_{3}, \xi_{4}>0$ then a co-existence equilibrium is always unstable;

(v) If $\xi_{1} \leq 0, \xi_{2}<0, \xi_{1} \xi_{2}<\xi_{3}, \xi_{4}>0$ then a co-existence equilibrium is either (a) always unstable, or (b) stable in a range such as $\left(\delta_{0}^{1}, \delta_{1}^{1}\right)$ and unstable in $\left(0, \delta_{0}^{1}\right) \cup\left(\delta_{1}^{1}, \infty\right)$.

\subsection{1 $k$ Very Large, $(m+h) r_{1}>p_{1}$}

This case is covered by Theorem 4(v)(b) so there is a unique co-existence equilibrium with susceptible prey, infected prey and predators present. From (4.4) the limiting value of $x^{*}$ as $k \rightarrow \infty$ is the unique root of

$$
\left(h-x^{*}\right)\left(m+x^{*}\right)\left(r_{1}\left(m+h-x^{*}\right)-p_{1}\right)-\left(\left(c_{1}+\gamma_{1} m\right)+\gamma_{1} x^{*}\right)\left(m+h-x^{*}\right) x^{*}
$$

in the interval $\left[0, \min \left[h, m+h-\frac{p_{1}}{r_{1}}\right]\right]$. Hence

$$
a_{11}=\frac{r_{1}}{k} \bar{S}=\frac{r_{1}}{k} \frac{\left(c_{1}+\gamma_{1} m\right)+\gamma_{1} x^{*}}{\left(m+x^{*}\right)} \rightarrow 0 \quad \text { as } k \rightarrow \infty .
$$

So as $k \rightarrow \infty$ the limiting values of $\xi_{1}, \xi_{2}, \xi_{3}$ and $\xi_{4}$ are

$$
\begin{aligned}
\xi_{1} & \rightarrow-a_{12} \bar{Y}-A<0, \\
\xi_{2} & \rightarrow-\left[a_{12}\left(\bar{Y}-\frac{\bar{S}}{h}\right)+A\left(1-\frac{x^{*}}{h}\right)\right], \\
& =-\frac{\left[a_{12} \bar{I}+A\left(h-x^{*}\right)\right]}{h}<0, \\
\xi_{3} & \rightarrow \bar{S} \bar{I}+A a_{12} \bar{Y}>0, \\
\xi_{4} & \rightarrow-\frac{A x^{*} \bar{S}}{h}+\frac{\bar{I} \bar{S} a_{12}}{h}+\bar{S} \bar{I} .
\end{aligned}
$$


To determine the sign of the limiting value of $\xi_{4}$ as $k \rightarrow \infty$ we note that

$$
\begin{aligned}
\bar{I}>\frac{A x^{*}}{h} \quad \text { if and only if } & h>\frac{c_{1} x^{*} \bar{Y}}{(m \bar{Y}+\bar{I})^{2}}, \\
& \text { if and only if } \quad h \bar{Y}>\frac{c_{1} x^{*}}{\left(m+x^{*}\right)^{2}} .
\end{aligned}
$$

But this will always be true as

$$
h \bar{Y}=\bar{Y}\left(h-x^{*}\right)+x^{*} \bar{Y}>\bar{Y}\left(h-x^{*}\right)>\frac{c_{1}}{m+x^{*}}>\frac{c_{1} x^{*}}{\left(m+x^{*}\right)^{2}}
$$

using (4.3). So in this case $\lim _{k \rightarrow \infty} \xi_{4}>0$ and by cases (iv) and (v) above in general apart from degenerate parameter values the co-existence equilibrium is either always unstable, or stable in a range such as $\left(\delta_{1}^{0}, \delta_{1}^{1}\right)$ and unstable in $\left(0, \delta_{0}^{1}\right) \cup\left(\delta_{1}^{1}, \infty\right)$.

\subsubsection{The Inequality $\xi_{4} \geq 0$}

Motivated by the example discussed above we may ask whether it is always the case that $\xi_{4} \geq 0$. However this is not always the case as the following counterexample shows:

Counterexample 5 It is possible to have a co-existence equilibrium with $\xi_{4}<$ 0 .

Proof. Choose $p_{1}=0, k m=c_{1}+\gamma_{1} m+\epsilon$, where $\epsilon$ is very small. Also choose $h$ very large in comparison with $m$. Then again we are in case (v)(b) of Theorem 4 and there is a unique co-existence with susceptible prey, infected prey and predators present. As $a_{3}$ is proportional to $\epsilon$ it is very small, so $x^{*}$ is very small and we have

by (4.3). As $a_{12}=0$

$$
x^{*} \approx 0, \quad \bar{Y} \approx \frac{c_{1}+\gamma_{1} m}{h m}
$$

$$
\begin{aligned}
\xi_{4} & =a_{11}(\bar{I}-A)+\bar{S}\left(\bar{I}-\frac{A x^{*}}{h}\right) \\
& =\frac{r_{1} \bar{S}}{k}(\bar{I}-A)+\bar{S}\left(\bar{I}-\frac{A x^{*}}{h}\right) \\
& =\frac{r_{1} \bar{S} \bar{I}}{k}\left(1-\frac{c_{1} \bar{Y}}{(m \bar{Y}+\bar{I})^{2}}\right)+\bar{S} \bar{I}\left(1-\frac{c_{1} x^{*} \bar{Y}}{h(m \bar{Y}+\bar{I})^{2}}\right) \\
& =\frac{r_{1} \bar{S} \bar{I}}{k}\left(1-\frac{c_{1}}{\bar{Y}\left(m+x^{*}\right)^{2}}\right)+\bar{S} \bar{I}\left(1-\frac{c_{1} x^{*}}{h \bar{Y}\left(m+x^{*}\right)^{2}}\right) .
\end{aligned}
$$

Substituting in the approximate values of $x^{*}$ and $\bar{Y}$ from (5.7) we deduce that

$$
\xi_{4} \approx \bar{S} \bar{I}\left(\frac{r_{1}}{k}\left(1-\frac{c_{1} h}{\left(c_{1}+\gamma_{1} m\right) m}\right)+1\right) .
$$

Hence $\xi_{4}$ is negative if $h$ is large in comparison with $m$. Therefore it is possible to have a co-existence equilibrium with $\xi_{4}<0$. 


\section{Hopf Bifurcation Around the Possible Co-ex- isting Equilibria $\bar{E}_{4}$ and $\bar{E}_{5}$}

By considering the diagrams giving the stability of the co-existence equilibria for various values of $\xi_{1}, \xi_{2}, \xi_{3}$ and $\xi_{4}$ as $\delta_{1}$ increases, we see that at points where the stability changes (either from stable to unstable or from unstable to stable) it is the constraint $e_{1} e_{2}>e_{3}$ that changes rather than $e_{1}>0$ or $e_{3}>0$. To formalise this as a theorem:

Theorem 6 Recall that $\delta_{1}$ is the ratio of $\delta$, the per capita growth rate of the pelicans, to $\lambda$, the prey disease transmission coefficient. Suppose that $\xi_{4} \neq 0$. As $\delta_{1}$ increases the co-existence equilibrium can change stability only as $\delta_{1}$ passes through a positive root of the quadratic equation in $\delta_{1}$

$$
e_{1}\left(\delta_{1}\right) e_{2}\left(\delta_{1}\right)-e_{3}\left(\delta_{1}\right)=0 .
$$

Proof. To prove this algebraically note that if $\xi_{4}<0$ then a co-existence equilibrium is always unstable so we can assume that $\xi_{4}>0$ and the constraint $e_{3}>0$ is always satisfied.

(i) If $\xi_{1} \geq 0$ then the constraint $e_{1}>0$ is always satisfied so as $\delta_{1}$ increases the stability of the co-existence equilibrium can change only as $\delta_{1}$ passes through one of the (at most) two possible roots of (6.1).

(ii) If $\xi_{1}<0$ then for $\delta_{1}<-\xi_{1}$ the co-existence equilibrium is unstable. At $\delta_{1}=-\xi_{1}$,

$$
e_{1}\left(\delta_{1}\right) e_{2}\left(\delta_{1}\right)=0<e_{3}\left(\delta_{1}\right)
$$

so the co-existence equilibrium is still unstable. For $\delta_{1} \geq-\xi_{1}$ the coexistence equilibrium will again change stability only as $\delta_{1}$ passes through one of the at most two possible positive roots of (6.1) for $\delta_{1}$.

We investigate the Hopf bifurcation for the system (2.1), taking $\delta_{1}$ as the bifurcation parameter. Suppose that $\omega\left(\delta_{1}\right)$ is an eigenvalue of the stability matrix at a co-existence equilibrium $\bar{E}_{4}$ or $\bar{E}_{5}$. Then Hopf bifurcation will occur if there exists a $\delta_{1}=\bar{\delta}_{1}$ such that

(i) $e_{1}\left(\delta_{1}\right) e_{2}\left(\delta_{1}\right)=e_{3}\left(\delta_{1}\right)$ with $e_{1}\left(\delta_{1}\right), e_{2}\left(\delta_{1}\right), e_{3}\left(\delta_{1}\right)>0$.

(ii) $\frac{d}{d \delta_{1}}\left(\left.\operatorname{Re}\left(\omega\left(\delta_{1}\right)\right)\right|_{\delta_{1}=\bar{\delta}_{1}} \neq 0\right.$.

Clearly (5.6) has two purely imaginary roots if and only if $e_{1} e_{2}=e_{3}$ for some value of $\delta_{1}$ (say $\left.\delta_{1}=\bar{\delta}_{1}\right)$ and $e_{2}\left(\bar{\delta}_{1}\right)>0$. There are at most two positive values of $\bar{\delta}_{1}$ at which we have Hopf bifurcation. For $\delta_{1}=\bar{\delta}_{1}$ we have

$$
\left(\omega^{2}+e_{2}\right)\left(\omega+e_{1}\right)=0
$$


which has three roots

$$
\omega_{1}=i \sqrt{e_{2}}, \quad \omega_{2}=-i \sqrt{e_{2}}, \quad \omega_{3}=-e_{1} .
$$

Thus in a neighbourhood of $\bar{\delta}_{1}$ the characteristic equation (5.5) cannot have real positive roots. In such a neighbourhood the roots have the form

$$
\omega_{1}\left(\delta_{1}\right)=u\left(\delta_{1}\right)+i v\left(\delta_{1}\right), \omega_{2}\left(\delta_{1}\right)=u\left(\delta_{1}\right)-i v\left(\delta_{1}\right), \omega_{3}\left(\delta_{1}\right)=-\phi\left(\delta_{1}\right)
$$

where $\phi\left(\bar{\delta}_{1}\right)>0$.

To apply the Hopf Bifurcation Theorem we have to verify the transversality condition

$$
\left.\frac{d}{d t}\left(\operatorname{Re}\left(\frac{d \omega_{k}}{d \delta_{1}}\right)\right)\right|_{\delta_{1}=\bar{\delta}_{1}} \neq 0, \quad k=1,2 .
$$

Substituting $\omega_{1}\left(\delta_{1}\right)=u\left(\delta_{1}\right)+i v\left(\delta_{1}\right)$ into (5.6), equating real and imaginary parts and calculating the derivative, we get

$$
\begin{aligned}
& R\left(\delta_{1}\right) u^{\prime}\left(\delta_{1}\right)-S\left(\delta_{1}\right) v^{\prime}\left(\delta_{1}\right)+T\left(\delta_{1}\right)=0, \\
& S\left(\delta_{1}\right) u^{\prime}\left(\delta_{1}\right)+R\left(\delta_{1}\right) v^{\prime}\left(\delta_{1}\right)+U\left(\delta_{1}\right)=0,
\end{aligned}
$$

where

$$
\begin{aligned}
& R\left(\delta_{1}\right)=3\left(u\left(\delta_{1}\right)\right)^{2}+2 e_{1}\left(\delta_{1}\right) u\left(\delta_{1}\right)+e_{2}\left(\delta_{1}\right)-3\left(v\left(\delta_{1}\right)\right)^{2}, \\
& S\left(\delta_{1}\right)=6 u\left(\delta_{1}\right) v\left(\delta_{1}\right)+2 e_{1}\left(\delta_{1}\right) v\left(\delta_{1}\right), \\
& T\left(\delta_{1}\right)=u^{2}\left(\delta_{1}\right) e_{1}^{\prime}\left(\delta_{1}\right)+e_{2}^{\prime}\left(\delta_{1}\right) u\left(\delta_{1}\right)+e_{3}^{\prime}\left(\delta_{1}\right)-e_{1}^{\prime}\left(\delta_{1}\right)\left(v\left(\delta_{1}\right)\right)^{2}, \\
& U\left(\delta_{1}\right)=2 u\left(\delta_{1}\right) v\left(\delta_{1}\right) e_{1}^{\prime}\left(\delta_{1}\right)+e_{2}^{\prime}\left(\delta_{1}\right) v\left(\delta_{1}\right) .
\end{aligned}
$$

At $\delta_{1}=\bar{\delta}_{1}, u\left(\bar{\delta}_{1}\right)=0$ and $v\left(\bar{\delta}_{1}\right) \neq 0$. If $S=0$ then $e_{1}\left(\bar{\delta}_{1}\right)=0$ which implies that $e_{3}\left(\bar{\delta}_{1}\right)=0$ which is a contradiction. Hence $S \neq 0$.

If $S U+R T \neq 0$ at $\delta_{1}=\bar{\delta}_{1}$, then

$$
\left.\frac{d}{d t}\left(\operatorname{Re}\left(\frac{d \omega_{k}}{d \delta_{1}}\right)\right)\right|_{\delta_{1}=\bar{\delta}_{1}}=-\left.\frac{S U+R T}{R^{2}+S^{2}}\right|_{\delta_{1}=\bar{\delta}_{1}} \neq 0 .
$$

Now from equation $(6.2)$ at $\delta=\bar{\delta}_{1}$,

$$
\begin{aligned}
S U+R T & =2 e_{2}\left(e_{1} e_{2}^{\prime}+e_{2} e_{1}^{\prime}-e_{3}^{\prime}\right), \\
& =2 e_{2} \frac{d}{d \delta_{1}}\left(e_{1} e_{2}-e_{3}\right) .
\end{aligned}
$$

Suppose that $\xi_{2} \neq 0$. Write $Q(\delta)=e_{1} e_{2}-e_{3}$. Then the equation $Q\left(\delta_{1}\right)=0$ is the equation of a parabola. At $\bar{\delta}_{1}, e_{1} e_{2}=e_{3}$ and so $e_{2}\left(\bar{\delta}_{1}\right)>0$. The tangent to the parabola

$$
\left.\frac{d Q}{d \delta_{1}}\right|_{\delta_{1}=\bar{\delta}_{1}}
$$

will be either increasing or decreasing except at the vertex. Hence there will be a Hopf bifurcation at $\delta_{1}=\bar{\delta}_{1}$, unless $\delta_{1}$ is a repeated root of $Q\left(\delta_{1}\right)=0$. 
In the degenerate case $\xi_{2}=0$ we must have $\xi_{3}>0$ and $\xi_{4}>0$ for a stable equilibrium to be possible. If $\xi_{3} \neq \xi_{4}$ then $Q\left(\delta_{1}\right)$ is a line with non-zero slope and a root at

$$
\delta_{1}=\bar{\delta}_{1}=\frac{\xi_{1} \xi_{3}}{\xi_{4}-\xi_{3}} .
$$

Hence if $\xi_{3}, \xi_{4}$ and $\frac{\xi_{1} \xi_{3}}{\xi_{4}-\xi_{3}}$ are all strictly positive, there is a Hopf bifurcation. If $\xi_{3}=\xi_{4}$ then $Q\left(\delta_{1}\right)$ is a constant, $S U+R T=0$ and we cannot conclude anything.

This completes our mathematical analysis of the general predator-prey disease system. In the next sections we shall look at some special cases of this system. First of all we shall examine the system when there is no infection in the prey and the predator interacts with the susceptible prey only. Next we establish a condition for disease to die out in the full system with susceptible and infected prey and predators. Then we shall look at the system with disease in the prey but no predators. Following this we look at disease effects on predators in the absence of healthy prey. Next in Section 11 we shall verify some of our theoretical results using numerical simulation with realistic parameter values.

\section{Interaction of Healthy Prey with the Predator in the Absence of Infected Prey}

Putting $I \equiv 0$ in the system $(2.1)$, we get

$$
\begin{aligned}
& \frac{d S}{d t}=r_{1} S\left(1-\frac{S}{k}\right)-\frac{p_{1} Y S}{m Y+S}, \\
& \frac{d Y}{d t}=\delta_{1} Y\left(1-\frac{h Y}{S}\right) .
\end{aligned}
$$

As before the term $\frac{p_{1} Y S}{m Y+S}$ is interpreted as zero at $S=Y=0$.

Hence there are three equilibria, the origin $\widetilde{E}_{0}=(0,0), \widetilde{E}_{1}=(k, 0)$ and $\widetilde{E}_{2}=(\widetilde{S}, \widetilde{Y})$ where $\widetilde{S}=h \widetilde{Y}$ and $\widetilde{Y}=\frac{k}{r_{1} h}\left(r_{1}-\frac{p_{1}}{m+h}\right)$. The last equilibrium is feasible only for $r_{1}>\frac{p_{1}}{m+h}$.

\section{(i) Behaviour of the System around $\widetilde{E}_{0}$}

The stability matrix is not well-defined at the trivial equilibrium $\widetilde{E}_{0}$. However, it is straightforward to show that this equilibrium is unstable as described earlier in the paper.

(ii) Behaviour of the System around $\widetilde{E}_{1}$

The stability matrix of the system is given by

$$
\left(\begin{array}{cc}
-r_{1} & -p_{1} \\
0 & \delta_{1}
\end{array}\right)
$$


The characteristic equation has one positive root so $\widetilde{E}_{1}$ will be unstable.

(iii) Behaviour of the System around $\widetilde{E}_{2}$

The stability matrix of the system is given by

$$
\left(\begin{array}{cc}
\frac{p_{1} h}{(m+h)^{2}}-\frac{r_{1} \tilde{S}}{k} & -\frac{p_{1} h^{2}}{(m+h)^{2}} \\
\frac{\delta_{1}}{h} & -\delta_{1}
\end{array}\right) .
$$

The equilibrium $\widetilde{E}_{2}$ is LAS if $\delta_{1}+r_{1}>\frac{p_{1}(m+2 h)}{(m+h)^{2}}$ and unstable if $\delta_{1}+r_{1}<$ $\frac{p_{1}(m+2 h)}{(m+h)^{2}}$. Note that

(i) If $S(0)=0$ then $S(t)=Y(t)=0$ for all $t$ so the system approaches $\widetilde{E}_{0}$.

(ii) If $S(0)>0$ but $Y(0)=0$ then $Y(t)=0$ for all $t$ and $S(t) \rightarrow k$ as $t \rightarrow \infty$ so the system approaches $\widetilde{E}_{1}$.

It is tempting to conjecture:

Conjecture 7 If $S(0)>0$ and $Y(0)>0$ and $\delta_{1}+r_{1}>\frac{p_{1}(m+2 h)}{(m+h)^{2}}$ then the system approaches $\widetilde{E}_{2}$ for large times, i.e. $S(t) \rightarrow \widetilde{S}$ and $Y(t) \rightarrow \widetilde{Y}$.

\section{Natural Disease Control}

We may wish to naturally eliminate disease from the system. We note that the infected prey will be wiped out from the ecosystem if $\gamma$, the per capita death rate of the infected prey, exceeds $k \lambda$, where $k$ is the carrying capacity of the system. Under this condition the whole infected prey population disappears and so the disease will also vanish.

Proposition 8 If $k \lambda<\gamma$ then disease will disappear from the ecosystem.

Proof. Choose $\epsilon>0$ such that $(k+\epsilon) \lambda<\gamma$. By the proof of Proposition $1 \exists t_{0}$ such that $S \leq k+\epsilon$ for $t \geq t_{0}$. Then for $t \geq t_{0}$

$$
\begin{aligned}
\frac{d I}{d t} & =I\left(\lambda S-\frac{c Y}{m Y+I}-\gamma\right) \\
& \leq I(\lambda(k+\epsilon)-\gamma) .
\end{aligned}
$$

Hence $I \rightarrow 0$ as $t \rightarrow \infty$.

The possible equilibria are $\bar{E}_{0}$ and $\bar{E}_{1}$, both of which are always unstable, $\bar{E}_{2}$ (which exists if $\left.r_{1}>\frac{p_{1}}{m+h}\right), \bar{E}_{4}$ and $\bar{E}_{5}$. If

(i) $r_{1}>\frac{p_{1}}{m+h}$, 
(ii) $\gamma_{1}+\frac{c_{1}}{m}+\frac{k p_{1}}{r_{1}(m+h)}>k$ and

(iii) $\delta_{1}+r_{1}>p_{1} \frac{(m+2 h)}{(m+h)^{2}}$

then we conjecture that $\bar{E}_{2}$ is globally asymptotically stable (GAS).

Conjecture 9 If (i)-(iii) above are satisfied then the equilibrium $\bar{E}_{2}$ is GAS.

\section{Impact of Disease on Prey in the Absence of Predators}

Putting $Y \equiv 0$ in the system (2.1), we get

$$
\begin{aligned}
& \frac{d S}{d t}=r_{1} S\left(1-\frac{S+I}{k}\right)-S I, \\
& \frac{d I}{d t}=S I-\gamma_{1} I,
\end{aligned}
$$

where $S$ denotes healthy prey and $I$ denotes infected prey. There are three ecologically meaningful equilibria for this system: $\widehat{E}_{0}=(0,0), \widehat{E}_{1}=(k, 0)$ and $\widehat{E}_{3}=(\bar{S}, \bar{I})$, where $\bar{S}=\gamma_{1}$ and $\bar{I}=\frac{r_{1}\left(k-\gamma_{1}\right)}{k+r_{1}} . \widehat{E}_{3}$ is a feasible equilibrium if and only if $k>\gamma_{1}$.

It is easily shown that the equilibrium $\widehat{E}_{0}$ is unstable and the equilibrium $\widehat{E}_{1}$ is LAS if $k<\gamma_{1}$ and unstable if $k>\gamma_{1}$. If $k=\gamma_{1}, \widehat{E}_{1}$ is neutrally stable. The stability matrix at $\widehat{E}_{3}$ is given by

$$
\mathbf{J}=\left(\begin{array}{cc}
\frac{-r_{1} \bar{S}}{k} & \frac{-r_{1} \bar{S}}{k}-\bar{S} \\
\bar{I} & \bar{S}-\gamma_{1}
\end{array}\right) .
$$

This leads to the characteristic equation

$$
\omega^{2}+H \omega+(H+\bar{S}) \bar{I}=0, \text { where } H=\frac{r_{1} \bar{S}}{k} .
$$

Both roots of the characteristic equation (9.2) have negative real parts so $\widehat{E}_{3}=$ $(\bar{S}, \bar{I})$ is LAS when it exists.

It is tempting to conjecture that whatever the initial conditions the system tends to $\widehat{E}_{1}$ if $k \leq \gamma_{1}$ and to $\widehat{E}_{3}$ if $k>\gamma_{1}$.

Conjecture 10 Whatever the initial conditions of the system (9.1) where the predators are absent tends to $\widehat{E}_{1}$ if $k \leq \gamma_{1}$ and to $\widehat{E}_{3}$ if $k>\gamma_{1}$. 


\section{Disease Effects on Predators in the Absence of Healthy Prey}

It is straightforward to show that if there are no healthy prey then both the infected prey and predators die out.

Theorem 11 If there are no healthy prey then both the infected prey and the predators die out so the system approaches $E_{0}$.

Proof. On simplifying the system (2.1), by letting $S \equiv 0$, we get

$$
\begin{aligned}
\frac{d I}{d t} & =-\frac{c_{1} Y I}{m Y+I}-\gamma_{1} I, \\
\frac{d Y}{d t} & =\delta_{1} Y\left(1-\frac{h Y}{I}\right) .
\end{aligned}
$$

Clearly $I(t) \rightarrow 0$ as $t \rightarrow \infty$. Hence given $\epsilon>0 \exists t_{0}>0$ such that for $t \geq t_{0}, I \leq$ $\epsilon$. For $t \geq t_{0}$

$$
\frac{1}{Y} \frac{d Y}{d t} \leq \delta_{1}\left(1-\frac{h Y}{\epsilon}\right)
$$

so if $Y \geq \frac{2 \epsilon}{h}, \frac{d Y}{d t}<-\delta_{1}$. Hence there is $t_{1} \geq t_{0}$ such that for $t \geq t_{1}, Y \leq \frac{2 \epsilon}{h}$ so $I \rightarrow 0, Y \rightarrow 0$ as $t \rightarrow \infty$.

\section{Numerical Work}

We explored our theoretical result with numerical simulations. We took most of our base parameter values from the study of an eco-epidemiological model of pelicans at risk in the Salton Sea by Chattopadhyay et al. [12], $r=3.0$ per day, $k=45.0, \lambda=0.006$ per day, $\gamma=0.24$ per day, $c=0.05$ per day. Additionally we take $\delta=0.09$ per day and $p=0$.

For our first simulation we took parameters as above except $m=1.0$ and $h$ $=0.2$. With this set of parameter values the equilibria are

$$
\begin{gathered}
\bar{E}_{0}=(0.0,0.0,0.0), \bar{E}_{1}=(45.0,0.0,0.0), \bar{E}_{2}=(45.0,0.0,225.0) \\
\text { and } \bar{E}_{3}=(40.0,4.587,0.0) .
\end{gathered}
$$

Note that $p_{1}=0, c_{1}+\gamma_{1} m>k m$ and $m>h$. So in (4.6) $b_{1}>0$ and $b_{2}>0$, so there is no co-existence equilibrium. As the conditions (5.1) and (5.2) are satisfied $\bar{E}_{2}$ is LAS and the other equilibria are unstable. We performed a variety of simulations and each time whatever the starting value the system tended to $\bar{E}_{2}$ as time became large. Figure 1 shows a typical simulation with starting values $(S, I, Y)=(60,40,300)$.

For the second simulation we took the same parameters except $k=75, h=$ 0.04 and $m=5.0$. With these parameter values we find that $\bar{E}_{0}=(0.0,0.0,0.0)$, $\bar{E}_{1}=(75.0,0.0,0.0), \bar{E}_{2}=(75.0,0.0,1,875.0)$ and $\bar{E}_{3}=(40.0,30.435,0.0)$ are possible equilibria. For these parameters 


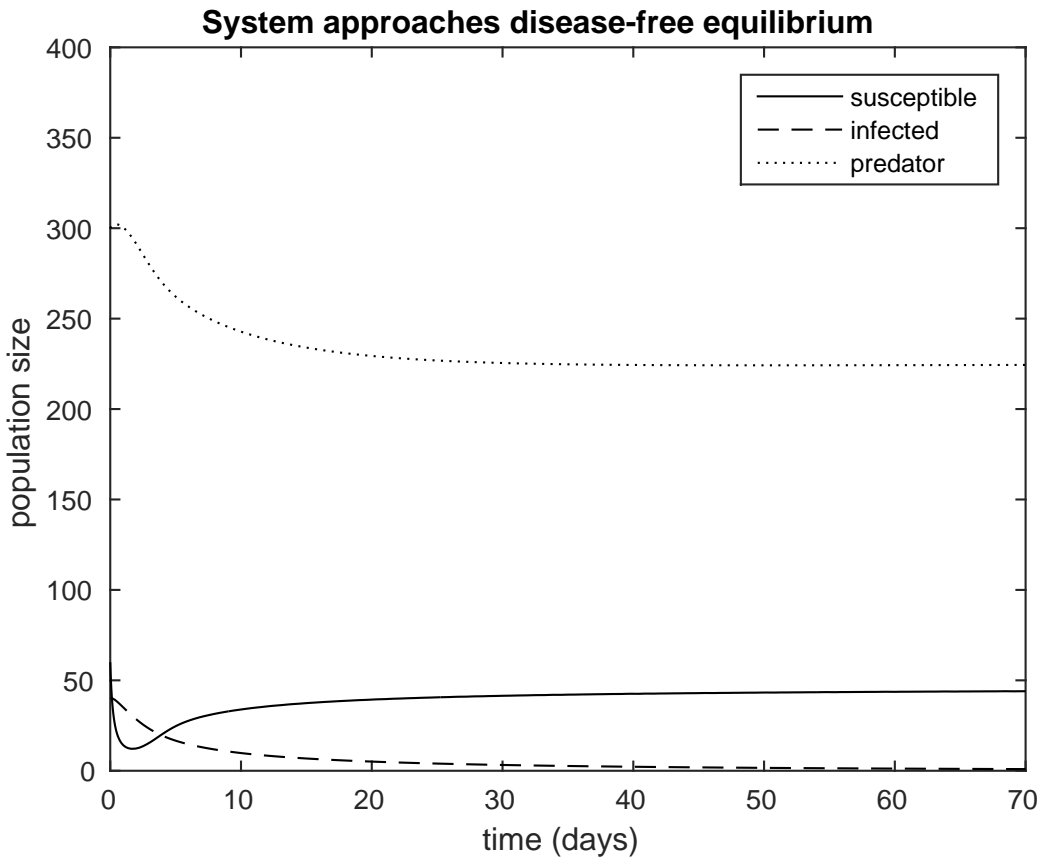

Figure 1: Simulation with $r=3$ per day, $k=45, \lambda=0.006$ per day, $\gamma=0.24$ per day, $c=0.05$ per day, $\delta=0.09$ per day, $h=0.2, p=0$ and $m=1.0$. Starting values $S=$ $60, I=40, Y=300$. System tends to $\bar{E}_{2}$. 


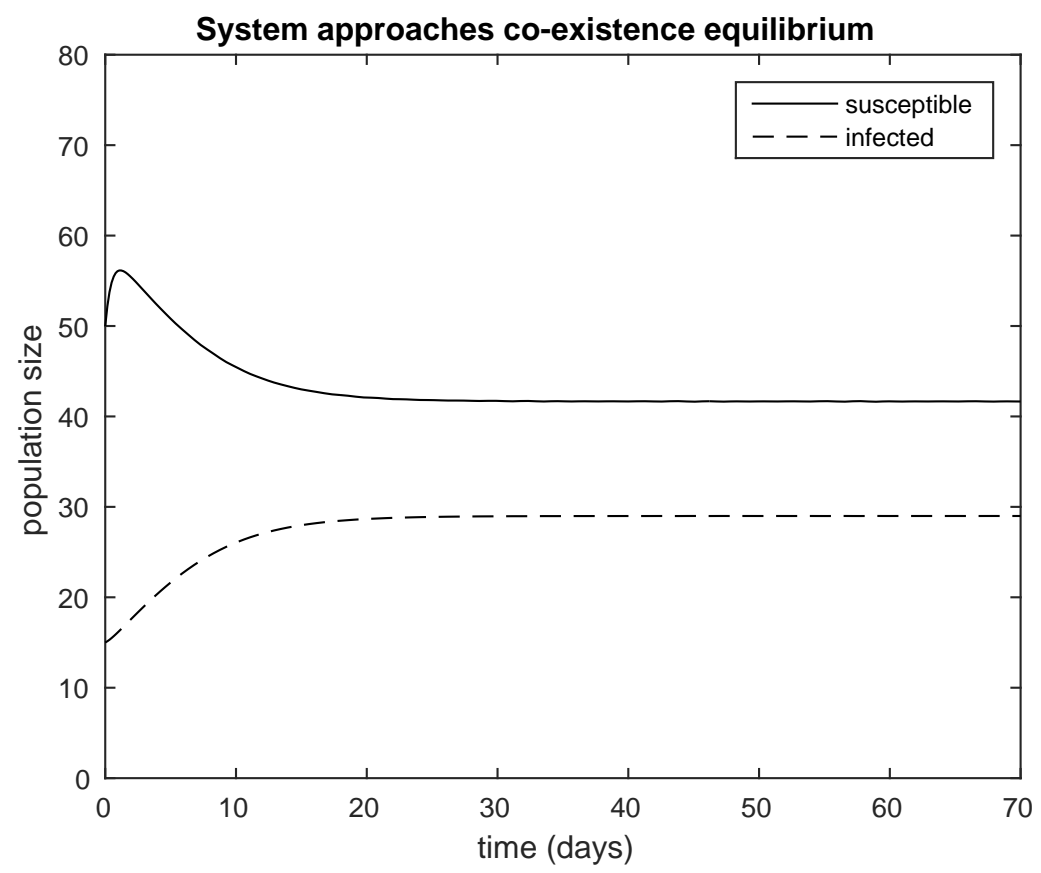

Figure 2: Simulation with parameter values as in Figure 1 except that $k=75, h=$ 0.04 . Starting values $S=50, I=15, Y=1,400$. System tends to unique co-existence equilibrium $\bar{E}_{4}$. Susceptible and infected prey.

$$
\gamma_{1}+\frac{c_{1}}{m}+\frac{k p_{1}}{r_{1}(m+h)}<k
$$

so the above four equilibria (including $\bar{E}_{2}$ ) are all unstable. $p_{1}=0$ and $c_{1}+$ $\gamma_{1} m<k m$ so there is a unique co-existence equilibrium. We calculate the unique root of equation (4.1) to be (approximately) $x^{*}=0.0164$ in this case and the corresponding co-existence equilibrium to be $(\bar{S}, \bar{I}, \bar{Y})=(41.7,29.0,1,766.0)$ (working only to three significant figures). We performed simulations with a range of starting values and found that whatever the initial value the system seemed to tend to the unique co-existence equilibrium. Figures 2 and 3 show a typical simulation with starting values $(S, I, Y)=(50,15,1,400)$. We have presented the simulation on two graphs, the first representing the tilipia and the second the pelicans.

To show that there could be two co-existence equilibria we took the parameter values to be the same as in Figure 1 except that $c=0.150006$ per day, $h=50$ and $m=5.0$. In this situation $p=0, \gamma_{1}<k, c_{1}+\gamma_{1} m>k m$ and $b_{1}<0$. Also the quadratic $\ell(x)$ given by (4.6) has two real roots, $x_{1}^{*}=0.08573$ and $x_{2}^{*}=0.01200$ (to four significant figures) in $[0, h]$. There are two corresponding co-existence equilibria $\bar{E}_{4}=(44.916,0.07715,0.8999)$ and $\bar{E}_{5}=(44.988,0.01080,0.9000)$.

To examine the existence of limit cycles arising by Hopf bifurcation we took 


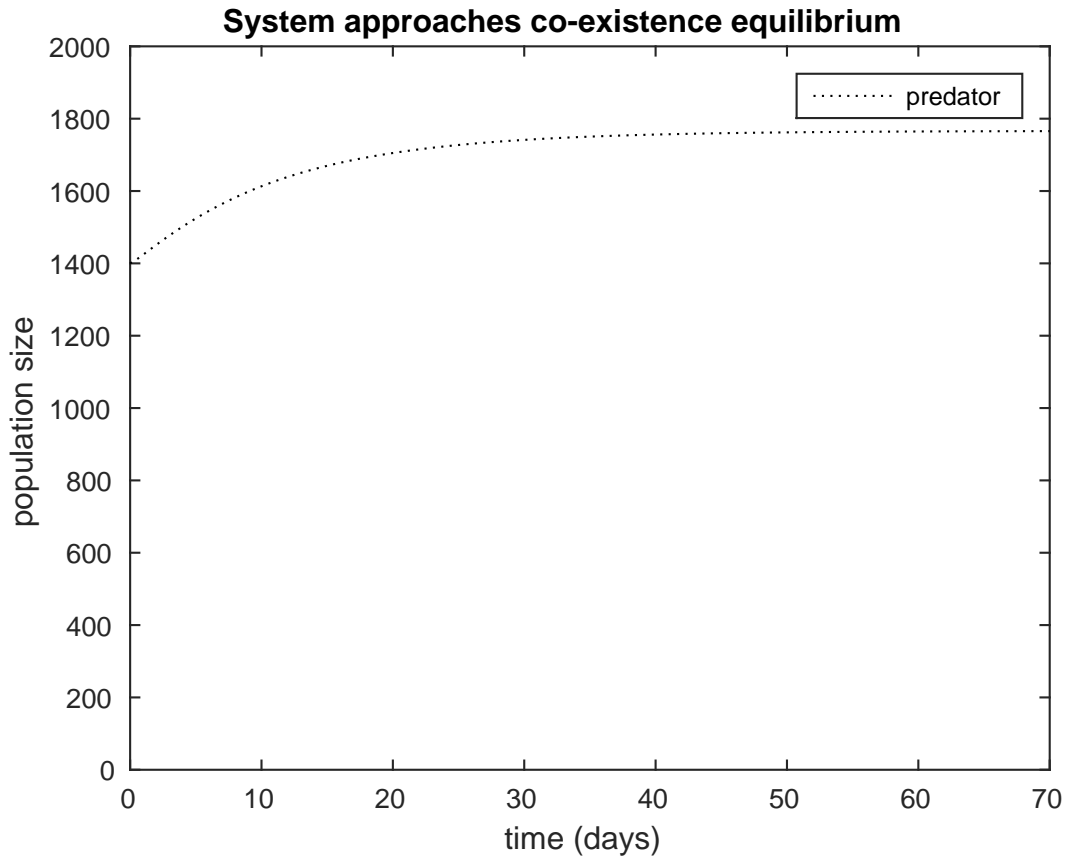

Figure 3: Simulation with parameter values as in Figure 1 except that $k=75, h=$ 0.04. Starting values $S=50, I=15, Y=1,400$. System tends to unique co-existence equilibrium $\bar{E}_{4}$. Predators. 


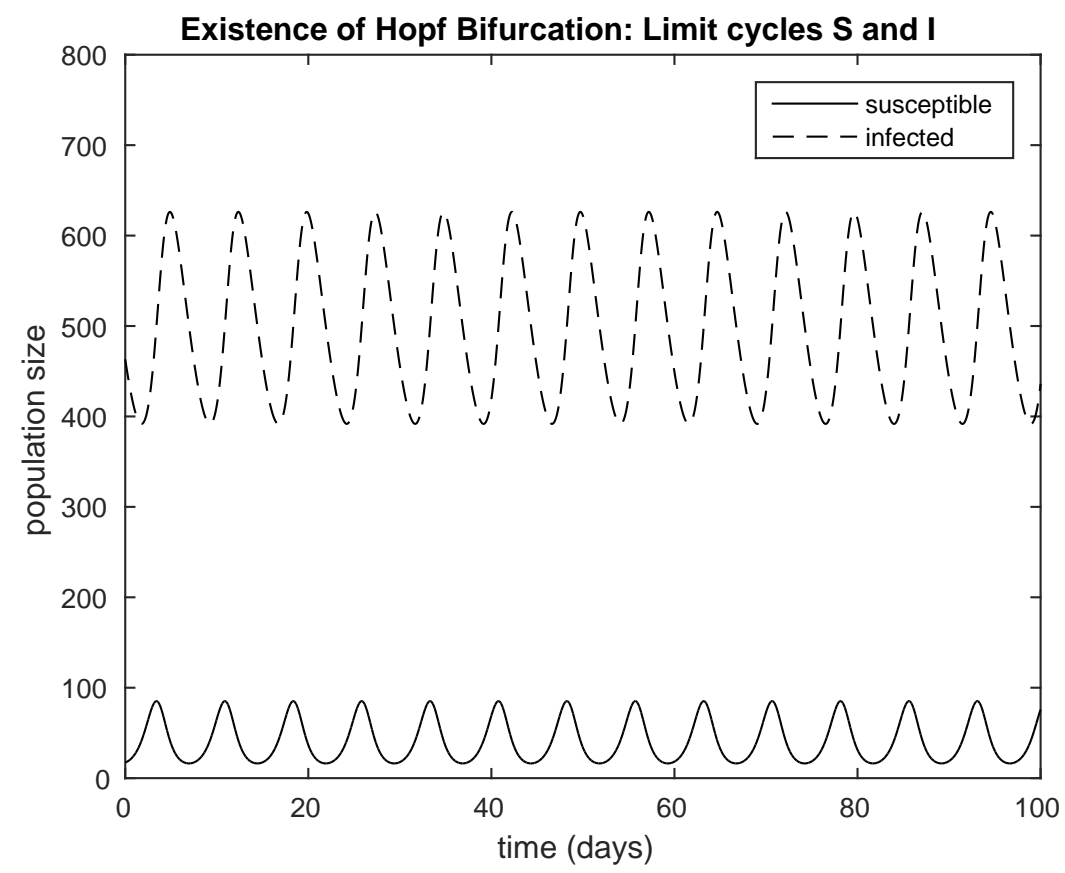

Figure 4: Simulation with parameter values and initial values as detailed in the text $(\delta$ $=0.09 /$ day) showing the existence of stable limit cycles about the unique co-existence equilibrium $\bar{E}_{4}$. Susceptible and infected prey.

$r=3.0 /$ day, $k=2,000,000, \lambda=0.006 /$ day, $\gamma=0.24 /$ day, $p=0, c=0.05 /$ day, $h=0.04$ and $m=5.0$. Again we have $p_{1}=0$ and $c_{1}+\gamma_{1} m<k m$ so there is a unique co-existence equilibrium (which is independent of $\delta$ ).

With these parameters we found $\xi_{1}=-0.00171432, \xi_{2}=0.00948071, \xi_{3}=$ $20,826.79$ and $\xi_{4}=20,826.34$. Examining the Routh-Hurwitz conditions we find that we expect the co-existence equilibrium to be unstable for $\delta \in\left[0, \delta_{0}\right)$ and stable for $\delta>\delta_{0}$ where $\delta_{0}=0.24911435$ to eight significant figures. Numerical simulations confirmed these results and showed that in the region $\left[0, \delta_{0}\right)$ stable limit cycles existed.

Figures 4 and 5 show the stable limit cycles for $\delta=0.09 /$ day in the susceptible and infected prey (Figure 4) and the predator (Figure 5) and Figures 6 and 7 with $\delta=100 /$ day show that the numbers of susceptible and infected prey (Figure 6) and the number of predators (Figure 7) tend to their steady-state equilibrium values $\bar{E}_{4}=(41.654,499.8646,13,537.98)$. In all of these figures the initial values were $S(0)=17.28, I(0)=463.13$ and $Y(0)=13,433.79$. For the parameter values for which we were able to show the existence of Hopf bifurcation the approach of the solution to the equilibrium value was very slow compared with the relatively fast transient oscillations. Hence it is not possible to show both on the same timescale and Figures 6 and 7 show the envelope of the solutions over a very long time which clearly shows that with this value of 


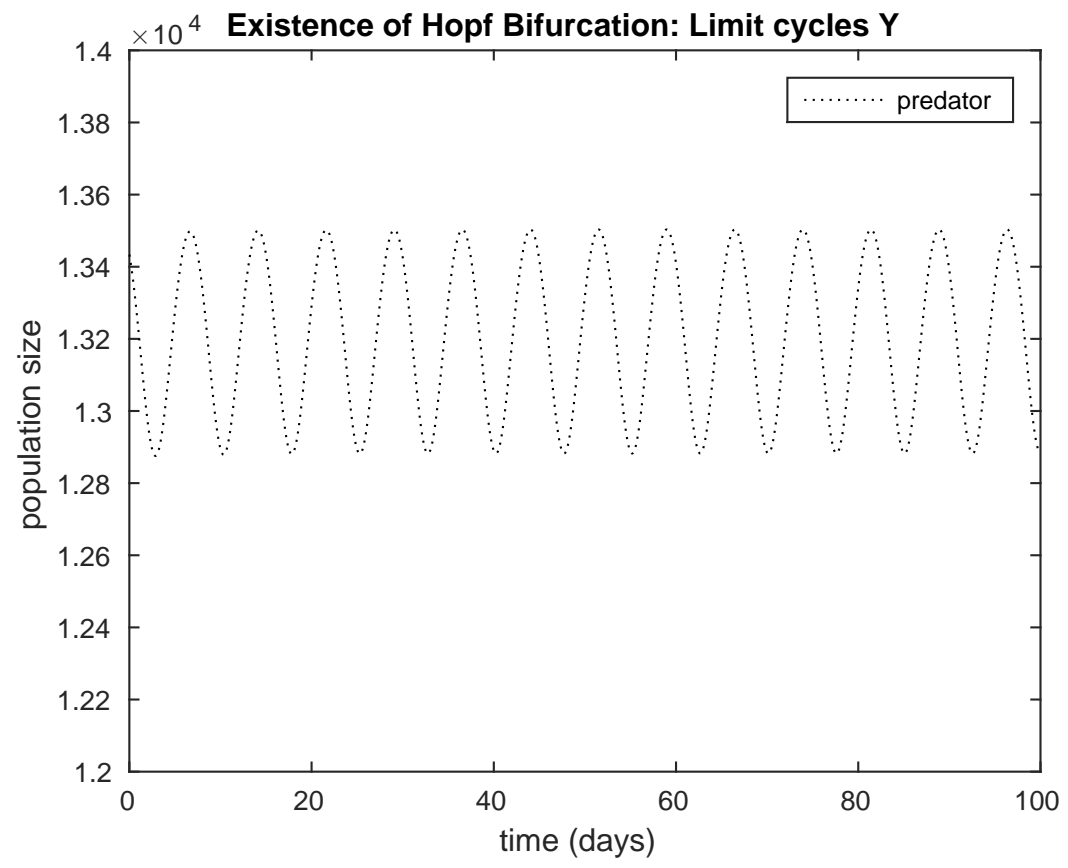

Figure 5: Simulation with parameter values and initial values as detailed in the text $(\delta$ $=0.09 /$ day) showing the existence of stable limit cycles about the unique co-existence equilibrium $\bar{E}_{4}$. Predators. 


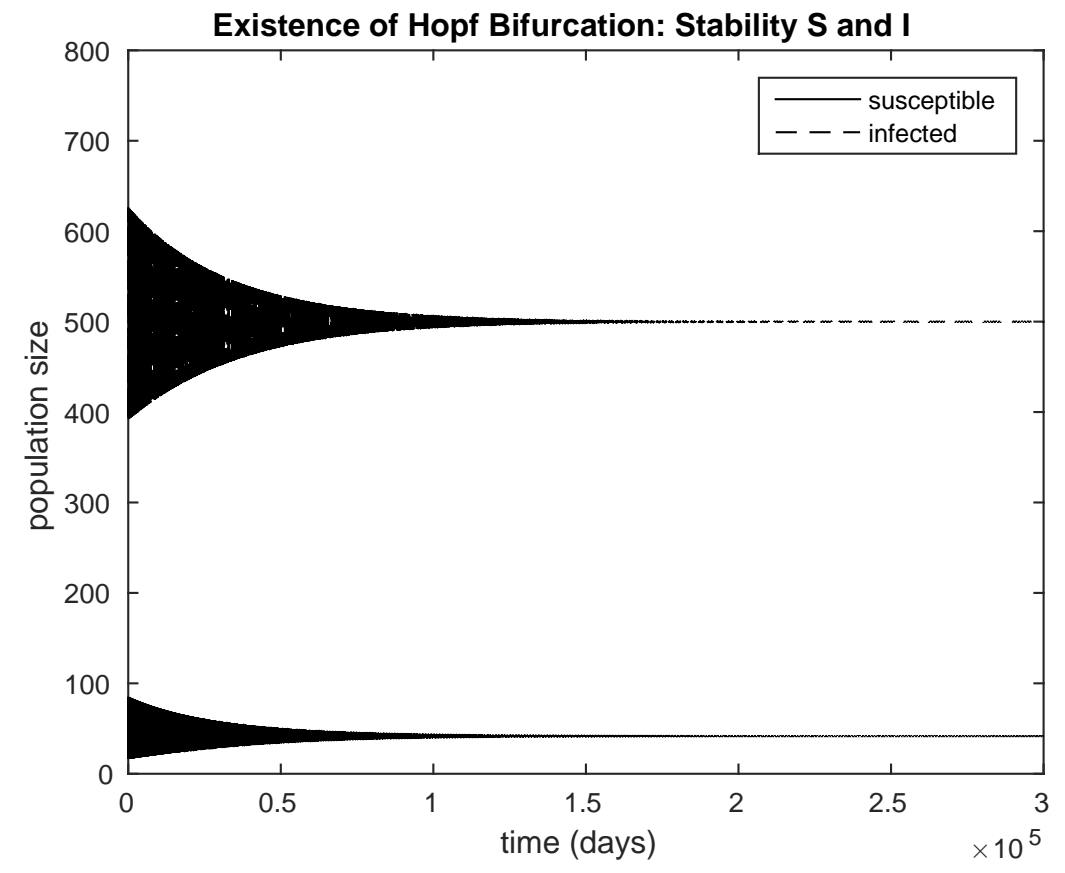

Figure 6: Simulation with parameter values and initial values as detailed in the text $(\delta=100.0 /$ day $)$ showing the system approaches the unique co-existence equilibrium $\bar{E}_{4}$. Susceptible and infected prey. 


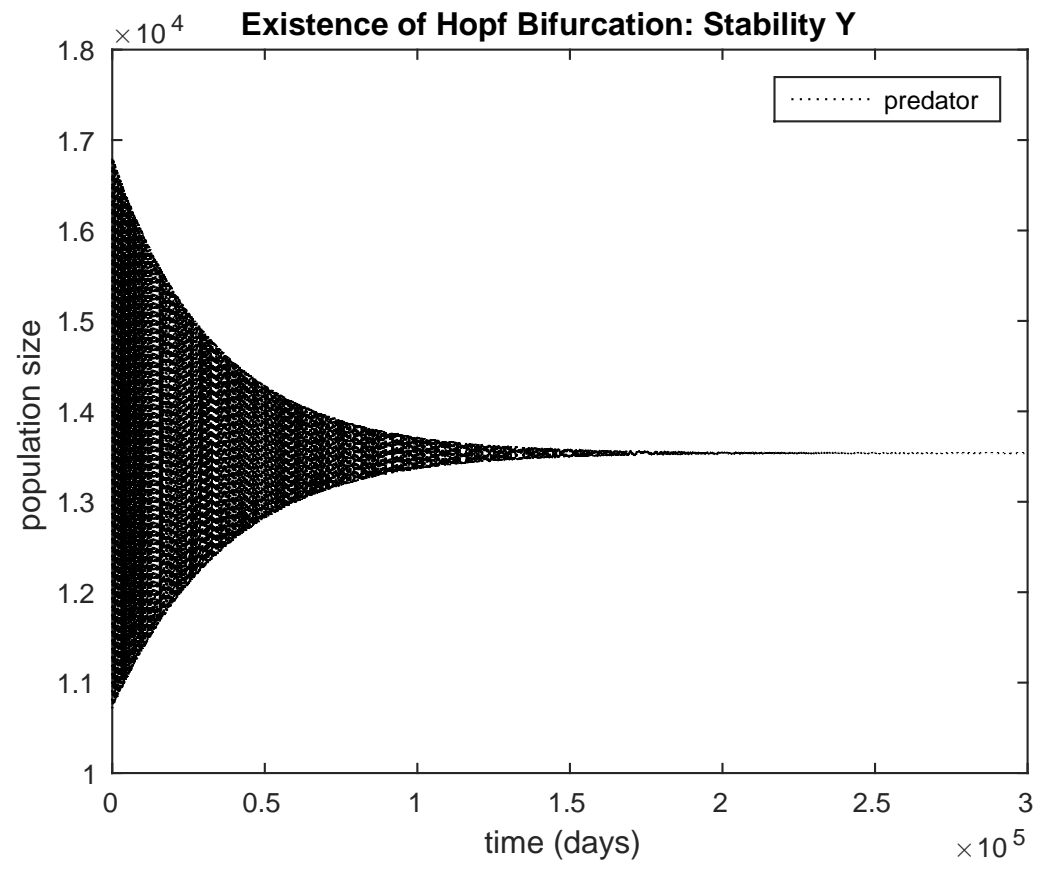

Figure 7: Simulation with parameter values and initial values as detailed in the text $(\delta=100.0 /$ day $)$ showing the system approaches the unique co-existence equilibrium $\bar{E}_{4}$. Predators. 


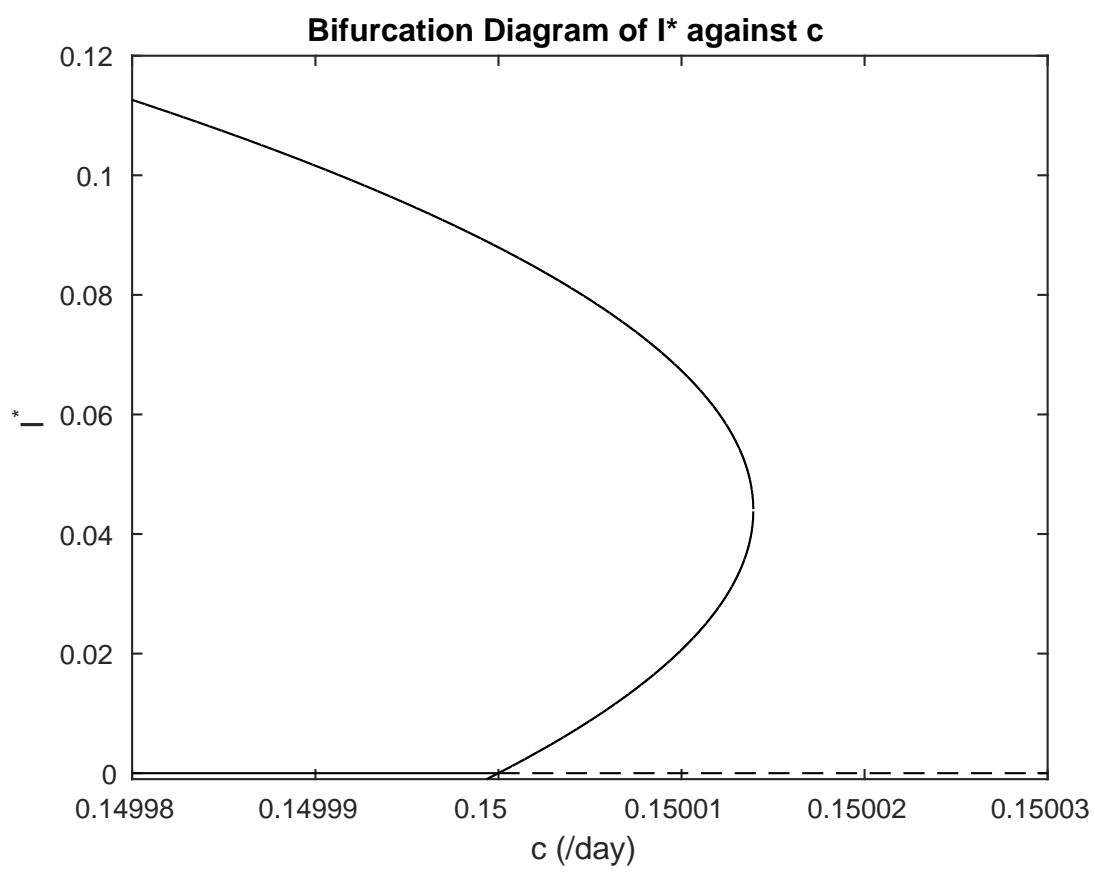

Figure 8: Bifurcation diagram of $I^{*}$ against $c$. Other parameters are as detailed in the text. A solid line indicates stability, a dashed line instability.

$\delta$ the solutions approach the unique co-existence equilibrium value.

To investigate the bifurcation structure and stability when multiple endemic equilibria exist we took $c$ as a bifurcation parameter. We really need a four dimensional bifurcation diagram but to understand the situation in a simple way we plot only the bifurcation diagram of $I^{*}$ against $c$. Hence the equilibria $\bar{E}_{0}, \bar{E}_{1}$ and $\bar{E}_{2}$ are all represented by $I^{*}=0$ on this bifurcation diagram. We took as parameters $r=3.0 /$ day, $k=45, \lambda=0.006 /$ day, $\gamma=0.24 /$ day, $p=0$, $\delta=0.09 /$ day, $h=50.0$ and $m=5.0$. With these parameters the unstable equilibrium $\bar{E}_{3}$ is represented by a constant line at $I^{*}=4.5872$. The susceptible prey and predator equilibrium $\bar{E}_{2}$ is stable for $c<0.15 /$ day and unstable if $c>$ $0.15 /$ day. For $c<0.15 /$ day there is a unique co-existence equilibrium which is stable. At $c=0.15 /$ day an additional stable co-existence equilibrium bifurcates in a forward direction from $\bar{E}_{2}$.

For 0.15 /day $<c<c_{0}$ where $c_{0}$ is approximately 0.1500139262 /day to eleven significant figures there are two co-existence equilibria but at $c=c_{0}$ they coalesce and disappear and for $c>c_{0}$ there do not appear to be any co-existence equilibria. Figure 8 shows the bifurcation diagram in the region $0.14998 /$ day $<c<0.15003 /$ day. 


\section{Summary and Discussion}

In this paper we have proposed and analysed a three-species eco-epidemiological model for the pelican-tilapia system in the Salton Sea. We modified the ratiodependent model of Greenhalgh and Haque [11] and considered that the susceptible and infected populations of tilapia are exposed to the predator to varying degrees for a more biologically realistic model. We showed that the system is bounded and found six equilibria. The first $\bar{E}_{0}$ was where all populations are extinct which is always unstable. The second $\bar{E}_{1}$ was where there is only susceptible prey which is also unstable. The third $\bar{E}_{2}$ was where there is no infected prey which exists if $r_{1}>\frac{p_{1}}{m+h}$. Conditions (5.1) to (5.4) for stability and instability of this equilibrium were derived. The fourth equilibrium $\bar{E}_{3}$ was where there are no predators. This is possible if $k>\gamma_{1}$ and is unstable. The fifth and sixth equilibria $\bar{E}_{4}$ and $\bar{E}_{5}$ were co-existence equilibria where both susceptible and infected prey co-existed with predators. We showed that for certain parameter values there could be either zero, one or two co-existence equilibria. Numerical simulations were used to confirm the analytical results. In addition we carried out theoretical analysis of Hopf bifurcation around the co-existence equilibria.

Next we moved on to some important special cases of the model where either the susceptible or infected prey or the predator was not present. If infection was not present in the prey then there were found to be three equilibria. The first, $\tilde{E}_{0}$, is trivial with neither susceptible prey or predators present and this is found to be unstable. There was also an equilibrium $\tilde{E}_{1}$ with the predator absent, which was also found to be unstable. There was a unique equilibrium $\tilde{E}_{2}$ with both predator and susceptible prey present, and conditions for stability of this equilibrium were found.

If the predators are not present then there are three equilibria present, $\hat{E}_{0}$, with neither infected nor susceptible prey present, which is always unstable, $\hat{E}_{1}$ with only susceptible prey present, which is LAS if $k<\gamma_{1}$ and unstable if $k>\gamma_{1}$. If $k>\gamma_{1}$ there is an additional equilibrium $\hat{E}_{3}$ with both susceptible and infected prey present which is always LAS. For the model with susceptible prey not present we showed that both the infected prey and the predators became extinct.

The results are more interesting than those of the original model by Greenhalgh and Haque [11] because in our current model the predator can consume susceptible prey as well as infected prey and both infected and susceptible prey can contribute to the carrying capacity. Thus our model is more general and realistic than the original model studied in [11]. As the model is more complex than the original model the potential behaviour is correspondingly more rich, diverse and interesting. For example an extra potential equilibrium $\bar{E}_{3}$ where there are no predators just susceptible and infected prey is possible in our model but not possible in the model studied in [11]. Additionally whilst the model of [11] always had a unique co-existence equilibrium in the current model there can be two co-existence equilibria simultaneously.

We have already discussed several other models of predator-prey systems 
with disease in the prey and pointed out the differences between them and our model. In particular none of these models uses a Leslie-Gower type equation for the predator growth.

Chattopadhyay and Arino [15] show that in their model the prey population ultimately tends to its carrying capacity and there are four equilibria: one where both the prey and the predator population have died out (like $\bar{E}_{0}$ ), one where the prey population is at its carrying capacity and both the disease and the predators have died out (like $\bar{E}_{1}$ ), one with both susceptible and infected prey and no predators (like $\bar{E}_{3}$ ) and a unique co-existence equilibrium (like $\bar{E}_{4}$ and $\bar{E}_{5}$ ). They discuss stability of the equilibria and show that limit cycles can arise from the stable co-existence equilibrium by Hopf bifurcation and discuss conditions for subcriticality and supercriticality.

The models discussed by Xaio and Chen [20], Haque and Chattopadhyay [16], Jin and Haque [17] and Pal and Samanta [21, 22] have only the four possible equilibria discussed above. Wang and Feng [23] discuss only the equilibrium which corresponds to $\bar{E}_{1}$ and the unique co-existence equilibrium: they do not establish whether other equilibria exist. All of these papers discuss conditions of stability of the equilibria and show that limit cycles can arise from the unique co-existence equilibrium by Hopf bifurcation.

The models of Arino et al. [18] and Kundu and Chattopadhyay [19] have all of the above equilibria and additionally an equilibrium with no infected prey only susceptible prey and predators. So also has the model of Rahman and Chakravarty [24]. Arino et al. give only a set of sufficient conditions for the co-existence equilibrium to exist and be unique, they do not discuss parameter values which do not satisfy this sufficient set of conditions. In the model of Kundu and Chattopadhyay [19] the general existence and uniqueness of the co-existence equilibrium is not discussed. Moreover Rahman and Chakravarty give two separate conditions for the uniqueness of each of (i) the equilibrium with only susceptible prey and predators and (ii) the co-existence equilibrium. They do not rule out multiple equilibria in either case if these conditions are not satisfied but on the other hand they do not show explicitly that multiple equilibria of either of these types is possible. They also discuss stability of possible equilibria and whether limit cycles can arise from the co-existence equilibrium by Hopf bifurcation.

Thus our model differs from most of the other models surveyed above in that the only one with a Leslie-Gower predator growth equation is Greenhalgh and Haque [11]. Our results differ from the results of most of these models in that in the majority of them the equilibrium corresponding to $\bar{E}_{2}$ does not exist and the co-existence equilibrium $\left(\bar{E}_{4}\right.$ or $\left.\bar{E}_{5}\right)$ is unique. The model of Rahman and Chakravarty [24] does not either show uniqueness of the co-existence equilibrium or demonstrate non-uniqueness. Thus our model is the first of these to explicitly demonstrate non-uniqueness of the co-existence equilibrium.

\section{Acknowledgements}

We are grateful to the referees for their helpful comments. We are also grateful to Sultan Qaboos University, Oman for financial support through a 
travel grant and Strathclyde University for financial support through an Interns@strathclyde internship for JSP for this project. DG is grateful to the Leverhulme Trust for support from Research Fellowship RF-2015-88.

\section{References}

[1] Anderson, R.M., May R.M., The population dynamics of microparasites and their invertebrate hosts, Philosophical Transactions of the Royal Society of London, Series B, Biological Sciences. 1981; 291 (1054):451-463. DOI: $10.1098 /$ rstb.1981.0005.

[2] Hadeler K.P., Freedman H.I., Predator-prey populations with parasitic infection, Journal of Mathematical Biology. 1989; 27:609-631. DOI: 10.1007/BF00276947.

[3] Gulland, F.M.D., Impact of infectious diseases on wild animal populations: a review. In: Grenfell, B.T., Dobson, A.P. (Eds.), Ecology of Infectious Diseases in Natural Populations, Cambridge University Press, Cambridge, 1995; pp.20-51. DOI: /10.1017/CBO9780511629396.002.

[4] Haque M., Venturino E., Increasing of the prey may decrease the healthy predator population in presence of a disease in the predator, HERMIS. $2006 ; 7: 38-59$.

[5] Kuang Y., Rich dynamics of Gause-type ratio-dependent predator-prey system, Fields Institute Communications. 1999; 21:325-337.

[6] Akcakaya H.R., Population cycles of mammals; evidence for ratiodependent predator-prey hypothesis, Ecological Monographs 1992; 62:119142. DOI: $10.2307 / 2937172$.

[7] Arditi, R., Ginzburg, L.R., Coupling in the predator-prey dynamics, ratiodependence, Journal of Theoretical Biology. 1989; 139 (3):311-326. DOI: 10.1016/S0022-5193(89)80211-5.

[8] Arditi, R., Ginzburg, L.R., Akcakaya H.R., Variation in the plankton densities among lakes; a case for ratio-dependent predation model, The American Naturalist. 1991; 138 (5):1287-1296. DOI: 10.1086/285286.

[9] Chattopadhyay, J., Bairagi, N., Pelicans at risk in Salton Sea an ecoepidemiological model, Ecological Modelling. 2001; 136 (2-3):103-112. DOI: 10.1016/S0304-3800(00)00350-1.

[10] Sarkar, R., Chattopadhyay J., Bairagi, N., Effects of environmental fluctuation on an eco-epidemiological model of the Salton Sea, Environmetrics. 2001; 12 (3):289-300. DOI: 10.1002/env.474. 
[11] Greenhalgh, D., Haque M., A predator-prey model with disease in the prey species only, Mathematical Methods in the Applied Sciences. 2007; 30:911929. DOI: $10.1002 / \mathrm{mma} .815$.

[12] Chattopadhyay, J., Srinivasu, P.D.N., Bairagi, N., Pelicans at risk in Salton Sea-an eco-epidemiological model-II, Ecological Modelling. 2003; 167 (12):199-211. DOI: 10.1016/S0304-3800(03)00187-X.

[13] Leslie, P. H., Some further notes on the use of matrices in population mathematics, Biometrika. 1948; 35 (3-4):213-245. DOI: 10.1093/biomet/35.34.213 .

[14] Leslie, P. H., A stochastic model for studying the properties of certain biological systems by numerical methods, Biometrika. 1958; 45 (1-2):1631. DOI: $10.1093 /$ biomet/45.1-2.16.

[15] Chattopadhyay J., Arino O., A predator-prey model with disease in the prey, Nonlinear Analysis: Real World Applications. 1999; 36 (6):747-766. DOI: 10.1016/S0362-546X(98)00126-6.

[16] Haque M., Chattopadhyay, J., Influences of non-linear incidence rate in an eco-epidemiological model of the Salton Sea, Nonlinear Studies. 2003; 10 (4):373-388.

[17] Jin Z., Haque M., Global stability of an eco-epidemiological model the Salton Sea, Journal of Biological Systems. 2006; 14 (3):373-386. DOI: 10.1142/S021833900600191X.

[18] Arino, O., El Abdlloui, A. Mikram, J. and Chattopadhyay, J., Infection in prey population may act as a biological control in ratio-dependent predatorprey models, Nonlinearity. 2004; 17 (3):1101-1116. DOI: 10.1088/0951$7715 / 17 / 3 / 018$.

[19] Kundu, K., Chattopadhyay, J., A ratio-dependent eco-epidemiological model of the Salton Sea, Mathematical Methods in the Applied Sciences. 2006; 29 (2):191-207. DOI: 10.1002/mma.671.

[20] Xiao Y., Chen L., A ratio-dependent predator-prey model with disease in the prey, Applied Mathematics and Computation. 2002; 131 (2-3):397-414. DOI: 10.1016/S0096-3003(01)00156-4.

[21] Pal, A. K., Samanta, G. P. Stability analysis of an eco-epidemiological model incorporating a prey refuge, Nonlinear Analysis: Modelling and Control. 2010; 15 (4):473-491.

[22] Pal, A. K., Samanta, G. P. A ratio-dependent eco-epidemiological model incorporating a prey refuge, Universal Journal of Applied Mathematics. 2013; 1 (2):86-103. DOI: 10.13189/ujam.2013.010208. 
[23] Wang, L., Feng, G. Stability analysis of a ratio-dependent predator-prey model incorporating a prey refuge, Journal of Applied Mathematics. 2014, Article ID 978578, 10 pages. DOI: 10.1155/2014/978758.

[24] Rahman, Md. S., Chakravarty, S., A predator-prey model with disease in prey, Nonlinear Analysis: Modelling and Control. 2013; 18 (2):191-209.

[25] Cosner, C., De Angelis, D.L., Ault, J.S., Olson, D.B., Effects of spatial grouping on the functional response of predators, Theoretical Population Biology. 1999; 56 (1):65-75. DOI: 10.1006/tpbi.1999.1414.

[26] Venturino, E., The influence of diseases on Lotka-Volterra systems, Rocky Mountain Journal of Mathematics. 1994; 24 (1):381-402. DOI:10.1216/rmjm/1181072471.

[27] Venturino E., Epidemics in predator-prey models; disease in the prey. In: Arino, O., Axelrod, D., Kimmel, M., Langlais, M. (Eds.), Theory of Epidemics. Mathematical Population Dynamics: Analysis of Heterogeneity, vol. 1, Wuerz, Winnipeg, 1995; pp.381-393.

[28] Haque M., Venturino E., The role of transmissible diseases in the HollingTanner predator-prey model, Theoretical Population Biology. 2006; 70 (3):273-288. DOI: 10.1016/j.tpb.2006.06.007.

[29] Xiao Y., Chen L., Modeling and analysis of a predator-prey model with disease in the prey, Mathematical Biosciences. 2001; 171 (1):59-82. DOI: 10.1016/S0025-5564(01)00049-9.

[30] Xiao Y., Chen L., Analysis of a three species eco-epidemiological model, Journal of Mathematical Analysis and Applications. 2001; 258 (2):733-754. DOI: $10.1006 /$ jmaa.2001.7514.

[31] Mukhopadhyay, B., Bhattacharyya, R., Role of predator switching in an eco-epidemiological model with disease in the prey, Ecological Modelling. 2009; 220 (7):931-939. DOI: 10.1016/j.ecolmodel.2009.01.016.

[32] Khan, Q.J.A., Greenhalgh, D., Hopf Bifurcation in epidemic models with a time delay in vaccination, IMA Journal of Mathematics Applied in Medicine and Biology. 1999; 16 (2):113-142. DOI: 10.1093/imammb16.2.113. 